\title{
Photobioreactors as a Dynamic Shading System Conceived for an Outdoor Workspace of the State Library of Queensland in Brisbane: Study of Daylighting Performances
}

\author{
Valerio R.M. Lo Verso, ${ }^{\mathrm{a}, *}$ Matin H.S. Javadi, ${ }^{\mathrm{b}}$ Simonetta Pagliolico, ${ }^{\mathrm{b}}$ Corrado Carbonaro, ${ }^{\mathrm{c}}{ }^{\text {Guido Sassi }}{ }^{\mathrm{b}}$ \\ a Department of Energy 'Galileo Ferraris', Politecnico di Torino, TEBE Research Group, corso Duca degli Abruzzi 24, 10129, Turin, Italy \\ ${ }^{b}$ Department of Applied Science and Technology Politecnico di Torino, corso Duca degli Abruzzi 24, 10129,Turin, Italy \\ ${ }^{c}$ Department of Architecture and Design DAD, corso Duca degli Abruzzi 24, 10129, Turin, Italy
}

\section{Article info}

\section{Article history:}

Received 8 October 2019

Revised 27 November 2019

Accepted 6 December 2019

Published online 9 December 2019

\author{
Keywords: \\ Photo-bioreactors \\ Microalgae \\ Responsive shading system \\ Visual comfort
}

\begin{abstract}
In the field of responsive shading systems, the use of photobioreactors (PBRs) containing microalgae seems to be a promising technology. Within this framework, this paper presents a case study where a PBR was specifically conceived as a shading system for an external workspace located on an open terrace of the State Library of Queensland (SLQ) in Brisbane. The proposed shading system makes use of PBRs as translucent screening elements, capable of regulating natural light to provide adequate visual comfort for a multi-purpose workspace, despite exposure of the site to direct sunlight and fluctuating weather conditions. The microalgae (Scenedesmus Obliquus) cultivated within the shading elements contribute to improving the environment by bio-sequestrating $\mathrm{CO}_{2}$ and producing oxygen. They are also a valuable raw material containing bioactive compounds with various applications including nutraceutical products, livestock feed, and biofuel synthesis. In addition, their green pigmentation is visually engaging and allows for the filtration of solar radiation. The paper focuses on analyzing the daylight performance in the outdoor workplace under the PBR shade. The biomass density of the PBR is adjustable across a wide range of light transmittances $(10 \%-80 \%)$ to counter the highly variable outdoor conditions. For different $\mathrm{T}_{\mathrm{v}}$ values, illuminance values were calculated using the simulation tool DIVA-for-Rhino and then analyzed as both point-in-time illuminance in June, September and December, and through climate-based daylight metrics such as the Daylight Autonomy and the Useful Daylight Illuminance. As a further step, the daylighting performances were compared to two other climates (Turin, Italy, and Dubai, United Arab Emirates), to verify which setting of the PBR may be suitable for different boundary conditions, thus making the system more widely applicable. The results in Brisbane showed that for clear sky conditions, higher levels of biomass saturation are needed $\left(\mathrm{T}_{\mathrm{v}}<20 \%\right)$ to remain in the 300-3000 lx 'optimal' illuminance comfort range, with lower concentrations $\left(\mathrm{T}_{\mathrm{v}}<30 \%\right)$ to remain within the 300-6000 lx 'acceptable' illuminance comfort range. Differently, in overcast sky conditions, the optimal range of illuminance is easily obtained with reduced levels of biomass saturation $\left(\mathrm{T}_{\mathrm{v}}<80 \%\right)$.
\end{abstract}

(C) 2019 The Author(s). Published by solarlits.com. This is an open access article under the CC BY license (http://creativecommons.org/licenses/by/4.0/).

\section{Introduction}

This paper presents a study on a novel photo-bioreactor (PBR) that was developed as a dynamic shading system in a real-world scenario: the Queensland University of Technology (QUT). Such a PBR combines the ability of green microalgae cultures to shield from direct sunlight by selectively absorbing the red radiation

\footnotetext{
${ }^{*}$ Corresponding author. Tel.: +390644585402, Fax.: 064880120

valerio.loverso@polito.it (V. R.M. Lo Verso)

matt.javadi@gmail.com (M. H.S. Javad)

simonetta.pagliolico@polito.it (S. Pagliolico)

corrado.carbonaro@polito.it (C. Carbonaro)

guido.sassi@polito.it (G. Sassi)
}

wavelengths (between $0.6-0.7 \mu \mathrm{m}$ ), with the capability of generating biomass rich in bioactive compounds. The PBR system has several promising functions from a sustainability standpoint: the carbon dioxide bio-sequestration and oxygen supply resulting from the photosynthesis performed by the microalgae (capable of improving the air quality of an indoor space); the production of biomass in indoor cultivation; and the enhancement of environmental comfort. The higher comfort level can be attributed to better air quality, an efficient shading from direct sunlight, and the visual appeal of the green surface, which has an impact on the psychological well-being of the occupants. For the particular real- 
world scenario, the PBR shading system was conceived for an outdoor multi-purpose workspace, located on the landscape roof terrace of the building.

This study stems from the DIGITAL (SUB) TROPICAL study of Carlorattiassociati (CRA) [1] for the Queensland government's 'Industry innovators in residence program'; Created as part of their International Education and Training Strategy which promotes collaboration with international thought-leaders, innovators, and key industry advisors to bring innovative ideas and insight to the local community. Within the program, one of the authors participated in a living lab program, along with colleagues and collaborators from the offices of CRA and the Senseable city lab (SCL) of the Massachusetts Institute of Technology (MIT), as well as along with other members from the QUT. The concept of the living lab was based on a systematic user co-creation approach, which integrated research and innovation processes.

The distinguishing innovation presented in this study is the development of a novel type of PBR panel, capable of combining the advantages of two different types of PBR systems: the flatpanel type and the tubular-raceway type. Even more importantly, the biomass density within the PBR was made adjustable through a specifically developed sensor-based control system, to provide a wide range of light transmittances $\mathrm{T}_{\mathrm{v}}$ (in the $10 \%-80 \%$ range). This huge range was needed to counter the highly variable outdoor conditions. In this respect, the entire PBR shading system is conceived as a responsive device.

The structural definition of the PBR system led to an exploration of novel design techniques and construction methods in concrete casting for complex geometries, making use of parametric design software to produce biomimetic forms reinforcing the theme of nature and organicism. Few cases exist on the use PBRs as static screening elements in architecture [2-5], which were used as references to support the development of the new proposal elaborated in this study. PBRs in architecture show great potential for positive environmental impact, and the cultivation of microalgae provides numerous benefits; not the least of which regards the bio-sequestration of $\mathrm{CO}_{2}$, the principal manmade contributor to the increase of greenhouse gases and global warming concerns [6]. A project by MIT's GreenFuel Technologies even goes as far as to make use of PBRs for the treatment of industrial exhaust gasses. Other advantages of microalgae cultivation include derivative products obtained from the resulting excess biomass such as, biofuels [7], pharmaceuticals, cosmetics [8], and nutraceutical compounds [9]. Furthermore, the implementation of PBRs in inhabited environments provides a means for the purification of indoor air and the treatment of wastewater [10], as well as aesthetic and wellness benefits in keeping with the concept of Biophilia.

\subsection{Goal of the study}

Within the above-mentioned framework, this paper presents a case study where a PBR shading system was developed as a preliminary design for the State Library of Queensland (SLQ) of the QUT in Brisbane. In more detail, the shading system was conceived as the cover for a multi-purpose workspace located on an outdoor terrace adjacent to the main library building.

The proposed shading system was conceived to be responsive, capable of regulating its light transmittance $\left(\mathrm{T}_{\mathrm{v}}\right)$ by adjusting the biomass density within the PBRs. This would allow adequate visual comfort levels to be provided for the occupants, despite the exposure of the site to direct sunlight and fluctuating weather conditions. The system's performance as a dynamic shading system was the main focus of the study, with the aim of exploring how the biomass density of the PBRs (and therefore the resulting $\mathrm{T}_{\mathrm{v}}$ values) would be able to contrast the variable sunlight and weather conditions in an outdoor workspace. In short, the main goals of the study were the following:

- To describe the application of a specifically designed PBR system to a real project in a real site.

- To assess the daylight performance of the outdoor space where the PBR system is meant to be installed as a responsive shading system.

\section{State of the art}

As an ongoing topic of debate and research, PBR technology shows a growing interest in both scientific and industrial communities, as demonstrated by the increasing number of scientific publications over the past forty years [11].

More and more investigations and projects have been focusing on the application of PBRs as elements in the built environment [2-5]. For the purposes of this study, a brief state of the art on technological advancement and scientific data in the field of PBRs was compiled in support of an informed design solution.

\subsection{Cultivation and grow rate of micro-algae}

Algae present in oceans, rivers and lakes are thought to make up around $10 \%$ of the total biomass of the world's plant-life; in spite of this, they are thought to be responsible for about half the planet's oxygen production. Although many types of algae are large (macroscopic) plants commonly known as seaweeds, there are also microalgae, tiny unicellular or colonial organisms that account for the majority of oxygen produced among all types of aquatic vegetation [12]. The same photosynthetic processes that are found in higher plants drive these microorganisms [13]. They can consist of bacteria, diatoms, other protists, and unicellular plants. However, unlike higher plants, microalgae are capable of absorbing nutrients directly, without the need for a vascular system [14]. Furthermore, they are able to convert $\mathrm{CO}_{2}$ into various raw materials depending on the species; which in turn has numerous applications in areas such as the production of biofuels, bioactive compounds, nutritional products, and livestock feed [1518]. This ability to absorb large quantities of $\mathrm{CO}_{2}$ provides an attractive alternative for the sequestration of $\mathrm{CO}_{2}$ emissions from industrial activities and has led to the conception of PBRs. Species considered among the main carbon sequesters are the Chlorococcum, Chlorella, Euglena and Scenedesmus genera [45,19-20].

The growth rate of microalgae in closed-system PBRs is the key parameter to their successful operation. This is affected by various factors and operating conditions [21]:

- Optical parameters, such as light intensity, exposure, spectral distribution, dark/light cycles, physical and geometric properties of the apparatus (i.e. the transparency of materials, and the ratio of irradiated surface area to reactor volume). Light capturing, distribution, and utilization are the key factors affecting biomass productivity and $\mathrm{CO}_{2}$ sequestration, as 
insufficient lighting can lead to inactivity and excessive lighting can lead to photo-inhibition and cell damage [21].

- $\mathrm{CO}_{2}$ : this is the carbon source for microalgae in autophototrophic culture and has a vital role in microalgal growth. Maintaining an optimal level of $\mathrm{CO}_{2}$ concentration is important as insufficient levels of $\mathrm{CO}_{2}$ limit growth, while oversaturation of $\mathrm{CO}_{2}$ could result in a low culture $\mathrm{pH}$, which would be inhibitory to some microalgal cells [21].

- Temperature: due to the space and lighting requirements of a commercial cultivation system, it will most likely be located outdoors and exposed to a wide range of day/night and seasonal temperature changes. In general, growth rates in microalgae cultures increase exponentially as temperatures increase, up to the point of optimal growth, after which the growth rate will decline. The optimal operating temperature for most species of microalgae is between $20^{\circ} \mathrm{C}$ and $30^{\circ} \mathrm{C}$, while growth rates are drastically reduced at temperatures of below $5^{\circ} \mathrm{C}$ and above $35^{\circ} \mathrm{C}[22]$.

- Heat transfer: this is necessary to maintain temperature in a proper range. A suitable strain of microalgae must be selected for different geographical situations [23], even if microalgae can grow under adverse environmental conditions (extreme temperature, $\mathrm{pH}$ and salinity) [19,24]. The opportunities for modulating the heat removal/supply are many (volume of reservoir, flow rates, parallel/series connection of shading elements, elements material (thermal conductivity). Even if the environmental condition changes the design choices allow the needs to be fulfilled.

- Acidity $(p H)$ levels: these are closely linked to both the temperature, and the level of $\mathrm{O}_{2}$ and $\mathrm{CO}_{2}$ in microalgae cultures. An optimal level of $\mathrm{pH}$ must be maintained for the cultivation of microalgae as excessive $\mathrm{pH}$ levels can lead to the disruption of cellular processes, resulting in complete culture collapse.

- Mass transfer (also called "culture mixing"): this is necessary to prevent the sedimentation of alga cells, to ensure appropriate levels of exposure to light and nutrients, to facilitate heat transfer, and to improve gas exchange. This is particularly important in high-cell-density microalgal cultures where inadequate mixing can result in drastically reduced transmission of light and increased levels of $\mathrm{O}_{2}$ [21].

- Cleanability and component maintenance: these considerations are necessary to avoid fouling (the formation of biofilm on reactor walls) and to maintain high levels of light transmission, as well as minimizing the chance of contamination. The main factors concerning cleanability are the smoothness of the internal surfaces, geometric factors such as bends and creases, and appropriate dimensioning of components in order to allow convenient cleaning [21].

Current challenges in the field of PBR technology, particularly for the production of biomass through the cultivation of microalgae, can be summarized as efficiency, scaling-up, and resilience [21]. The optimization of productivity and efficiency in PBR systems is crucial to the overall viability of the technology. Moving to large-scale production units poses a series of issues regarding the scalability of existing prototypes [25], while moving from controlled laboratory conditions to the irregular conditions of the natural environment challenges the resilience of the microbiological subjects. In addressing these issues, a global effort has yielded a wide variety of solutions, which have then been assessed, developed, and characterized.

\subsection{Photobioreactor design}

In comparison to open pond type systems, closed PBRs can support much higher levels of photosynthetic efficiency, biomass productivity, and biomass concentration [21]. Olivieri et al. [11] provide an overview of advances in PBR technology, investigating various configurations, operating strategies and applications. Based on tested configurations, closed PBR systems can be subdivided into tubular, vertical column, flat panel, and membrane type systems [11], each with inherent strengths and weaknesses. The principal focus of most research into PBR configuration has been the optimization of productivity and efficiency within the system with respect to the various parameters that affect microalgae growth. Most PBR systems elaborated to date fall under four main categories:

- Tubular PBRs are among the most popular configurations of PBRs [26]. Typically, they consist of an array of transparent tubes arranged in various configurations (e.g., straight, bent, or spiral). Tube diameters of $0.1 \mathrm{~m}$ or less are typically employed to ensure a high rate of biomass productivity. Tubular PBRs can be oriented in horizontal, inclined, and vertical arrangements. Horizontal and inclined configurations are able to provide a better angle for incident light compared to vertical tubular reactors, thus increasing the efficiency of light harvesting. On the other hand, this leads to the accumulation of heat, which could require the addition of expensive temperature control systems, presenting significant drawbacks when attempting to scale-up reactors [27]. Often, a form of heat exchanger is incorporated into the design to maintain an optimal growth temperature [28]. Tubular PBRs also achieve a greater surface-area-to-volume ratio when compared to vertical column systems due to their ability to evenly distribute their weight across an area. This as opposed to vertical column types, which must have a sufficient cross section to support the structure above them, leading to larger units with a lower surface area to volume ratio. In tubular PBRs, mixing is accomplished by circulating the culture medium, and the mass transfer coefficient can be increased by raising the system's rate-of-flow. This poses a risk however, as increased shear rates have been found to damage the alga cells [29].

- Rigid vertical column PBRs are usually cylinders with a radius of up to $0.2 \mathrm{~m}$ and heights of up to $4 \mathrm{~m}$. These columns must have a small radius to maintain an ideal surface-area-tovolume ratio. Vertical column PBRs are characterized by their high volumetric gas transfer coefficients. The bubbling of gas from the bottom of the column enables efficient $\mathrm{CO}_{2}$ utilization and $\mathrm{O}_{2}$ removal. The primary advantage of an airlift PBR is the excellent mixing it offers. This is due to the turbulence created by the rising air bubbles, which ensure that the cells are evenly exposed to light radiation, even with relatively large column diameters and higher biomass densities. In addition, the simplicity of the structure makes for convenient maintenance and cleanability. The main limiting factor in vertical column PBRs is light penetration, which is fundamental to growth rates, meaning the cross section diameter is limited in order to ensure optimal diffusion of light throughout the system [21]. 
- Flat panel PBRs consist of transparent plates typically arranged in vertical and inclined configurations, with high illuminated surface-to-volume ratios. These PBRs can be oriented to ensure optimal harvesting of solar energy, and the continuous surface of the panels results in an even distribution of light throughout the system. On the other hand, their drawbacks are limitations in culture flow control, and high costs of construction. The thickness of the plates is a fundamental aspect of flat panel PBR design as it affects both the light path, and the surface-area-to volume ratio. A lesser thickness allows for better diffusion and distribution of light, In general, the shorter the light path the higher the biomass productivity [30,31]. It is worth noting that thin PBRs are more expensive to make, more difficult to clean, and more readily prone to overexposure (photoinhibition) and temperature fluctuation [21].

- Plastic bag or membrane PBRs are low cost and have good sterility at start up, which makes them especially attractive for commercial scale production. These bags can also be fitted with aeration systems to improve yields. Large membrane PBRs of up to $2000 \mathrm{~L}$ were once popular in aquaculture operations for the cultivation of algae as livestock feed and are still in limited use today [32,33].

A further variation of vertical flat panel PBRs has been proposed, consisting of a disposable plastic bag positioned between two iron frames [34], with substantial cost reduction to PBR construction. Nevertheless, the disposal of used plastic bags may present a significant challenge in large-scale operations. The principal drawbacks to membrane PBRs, aside from being inherently fragile, are inadequate mixing and frequent "culture crashing" due to microbial contamination. It was also noted that the use of larger bag volumes in scaling-up the system does not necessarily lead to increased productivity [35].

Pagliolico et al. [4,5] demonstrated that transparent polyethylene, flexible hanging bags with small circular compartments, allowed the cultivation of Scenedesmus Obliquus for at least 21 days, under natural light/dark cycles, and at controlled indoor environmental temperature without the need for mixing.

\section{Case study: 'The Edge' of the State Library of Queensland (SLQ) in Brisbane}

\subsection{Description of 'The Edge' site}

The specific location of the project is a building known as 'The Edge', directly overlooking the Brisbane River and in-between the State Library of Queensland (SLQ) and the Queensland Art Gallery (Fig. 1). These two buildings have recently undergone transformations: The State Library was redeveloped in its current form by Donovan Hill [36] in Association with Peddle Thorp Architects, while The Edge was refurbished by M3 architects and developed into a creative resource hub and community makerspace. Intended to broaden and enhance the activities of the State Library, The Edge organization describes itself as "a model for the library of the future, a visionary space for 'creating creatives'; a melting pot of ideas, capacity-building, experimentation and innovation".

Recently, The Edge has been the subject of a series of enhancement proposals ranging from landscaping projects to digital installations and additional activities for the user community. One such proposal was the "Even Greener" strategic landscaping plan. Its goal was to improve the landscaping and green spaces of the cultural precinct as well as enhancing the water harvesting and energy generating capabilities, offsetting waste, and improving the overall sustainability of the area. Another project "The Green Screen" proposed the installation of largescale LED screens on the rooftop level for broadcasting internal activities to the surrounding area and across the river to the city center. The most important shared theme in the various proposals was an increasing emphasis on sustainability, which was adopted as a core element in the development of the concept for this study. The Edge is developed on three levels, with the first two levels housing various specialized facilities, and the upper level consisting of a landscaped rooftop terrace. The terrace currently functions as an events-space, and hosts a stepped concrete pavilion and a permanent installation by Anthony Pryor titled 'Approaching Equilibrium'. The entire upper level has a surface area of approximately $1400 \mathrm{~m}^{2}$ and shows great potential for the creation of an outdoor workspace; this would allow for an area of roughly $750 \mathrm{~m}^{2}$ to be used to improve and expand the activities already present in the surrounding areas.

The North-Eastern orientation of the site and the lack of significant obstructions ensures that the entire upper area of the terrace receives an abundance of natural light throughout the day. This constant exposure to direct sunlight however, would be unsuitable for the purposes of a work environment. Thus, a type of dynamic, responsive shading system was needed to achieve optimized and constant levels of comfort, despite the fluctuation of environmental conditions. Different types of responsive shading systems exist and were considered, for instance able to change their shape [37-38] or dynamically moving along the façade to change the shading effect over time [39]. A detailed review on typologies, system design, mechanism and controlling methods can be found in a study from Al-Masrani and K.M. AlObaidi [40]. Among several technologies, the use of PBRs was selected as the most suitable to shade the landscape terrace of the Library, due to its sustainability in terms of trade-off between aesthetical issues, environmental comfort assured in the space and final acceptance for the occupants.

\subsection{Needs and requirements of algae PBR as shading system for the rooftop terrace}

The city of Brisbane is known for its mild tropical climate, which provides an average of over 7 hours of sunlight per day. With mean temperatures ranging between $15^{\circ} \mathrm{C}$ and $25^{\circ} \mathrm{C}$, it shows great potential for the development of new types of outdoor workspaces. The relatively stable and temperate climate could also prove to be an ideal testing ground for the intensive cultivation of microalgae and practical applications of PBRs in architecture. Some recent studies estimate the optimal thermal range for operating PBR systems to be between $15^{\circ} \mathrm{C}$ and $25^{\circ} \mathrm{C}[4,5,22]$.

Various factors regarding the existing context were considered throughout the development of the project, which can be defined as three main areas of interest: 

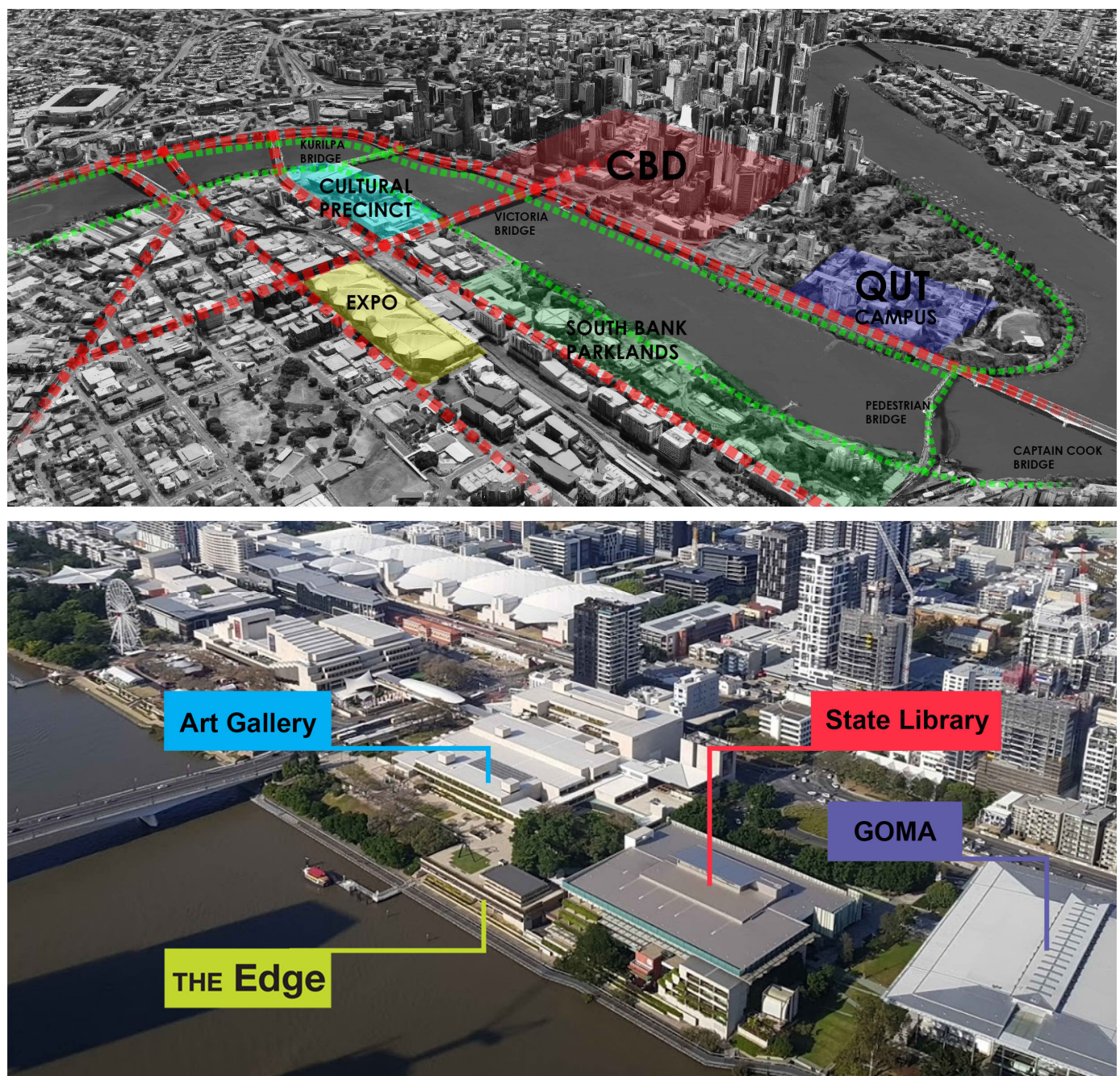

Fig. 1. Images of the urban context where the Edge is located.

- Social issues, concerning the users of the library facilities, their behavior in the existing spaces, and their needs. These issues were based on studies and on-site surveys, which provided insight into the behavior and characteristics of the users of the library facilities. According to the data gathered by abovementioned Hill of Arup Pty. Ltd. [36], patrons of the public library are predominantly under-30 years old, and frequent the library at least once a week to work exclusively on personal laptop computers. The survey also showed that $70 \%$ of users frequent the facilities in groups, $50 \%$ make use of the existing outdoor environment on the terrace, and $70 \%$ make use of the facilities outside opening hours. Aside from the survey, direct observations were made by one of the authors of this study, during a residency at the library facilities; a number of users were observed to be regularly present in external areas with Wi-Fi coverage from early hours of the morning until opening time. After closing, users were observed exiting the interior spaces of the library to once again make use of the available spaces of the atrium. In many cases, users were observed to find places to work outside the library building, and in quite uncomfortable conditions, due to a scarce availability of seating and utilities. Further observations of the behavioral patterns showed a high frequency of library users working in groups, often changing places within the group to engage other members, resulting in a noisier environment that could be disturbing to other library users. Additional improvements were considered in favor of security, access to toilets, food and drink, lockers and other such amenities. Such in-situ, direct observations served in the development of the architectural project guidelines by identifying key areas for improvement.

- Spatial issues, regarding the relationship between the project and its built context. The outlining of distinct activities and their general location within the project was informed by the direct observations mentioned above, and was considered fundamental to the development of a successful solution. A space was therefore described to host work-related activities for both individuals and groups, as well as observation and leisure activities. Consequently, the space can be generally divided into three zones; the first for individual and small groups; the second, for larger groups; and the third, as an observation deck taking full advantage of the site's unique location. Additional provisions such as restroom facilities and refreshments were also included in the final configuration of the site. This led to the definition of a general scheme of pathways that allowed users to move freely without disrupting the ongoing activities within the workspace.

- Environmental and climatic issues, addressing the projects climatic concerns as well as considerations regarding the 
management of resources. These issues include impermeability, rainwater harvesting, solar irradiance, wind conditions, and noise pollution among others. Rainwater harvesting is a fundamental part of the overall water management strategy and was taken into consideration when defining the form of the structure due to the significant amount of water used by the PBR system. The project included rainwater harvesting and greywater treatment in both the definition of the structure, and its relationship with the surrounding built environment. Likewise, solar irradiance is a key factor in the project, both in the form of incident heat on the exposed surfaces, which can greatly affect the overall efficiency of the system, and in terms of daylighting, which is crucial to the activities taking place within the space. Incoming winds, which could be a further disturbance to the work activities, were also taken into consideration in the definition of the structure by identifying prevailing wind patterns and taking provisions to ensure the space is adequately shielded.

Electric lighting was a further concern for the project. During the day, daylighting levels are controlled by the PBR shading system by adjusting its light transmittance $\left(\mathrm{T}_{\mathrm{v}}\right)$, while at night electric lighting is prescribed to maintain the target illuminance levels. Aside from the functional illumination required for the work environment, additional LED lighting was considered as a means to increase the visibility of the project, considering the exceptional position of the site. This vibrant lighting strategy was conceived in accordance with the planning authorities' current objectives and would make the project eligible for significant grants under the "light up Brisbane" initiative.

To conclude, the BPRs responsive shading system represents an architectural component able to improve on the one hand aspects such as the aesthetic and cultural values of the space on the building terrace, on the other hand its performance as a trade-off between daylighting conditions inside the indoor space, climatic zone and user requirements. Finally, the system described in this study is well aligned with the research line called 'biomimetic building skin', which is based on the integration of biology in architecture [41]. An example of such a research line is the system described by Dewidar et al. [42]: they presented a theoretical model for an adaptive wall system that can mimic nature called the Self-Active Bioclimatic Strategy (SABS). The system takes advantage of algae bio-reactor panels with a kinetic responsive behavior in such a way that it adapts to environmental demands to achieve energy efficiencies.

The architectural design of the PBR system also addressed a number of other concerns.

- Microalgae selection: various different aspects were considered in choosing the optimal microalgae for the project. Ranging from the physiological needs of the organism itself, to its efficiency in $\mathrm{CO}_{2}$ sequestration, light absorption capabilities, wastewater treatment capacity, tolerance of pollutants, and aptitude for biofuel extraction.

Scenedesmus Obliquus (S. Obliquus hereby) has shown to be adaptable for use in static shading elements [4,5] and is the species of microalgae capable of providing the lowest values of light penetration among freshwater microalgae with biofuel applications, making it an ideal candidate for use in a shading PBR device.
A review by Palmer [43] listed algae types in the order of their tolerance to organic pollutants as reported by 165 authors. The review included 60 genera and 80 species and ranked the Scenedesmus genera among the most resistant

- PBR type Selection: considering the installation as a permanent structure, exposed to the external environment and intended for public use, membrane type PBRs were considered unsuitable due to their fragility. The most common types of PBRs for horizontal applications are tubular and flat-panel systems. Flat panel PBRs prove to be more effective in vertical installations as their mass transfer systems are based primarily on airlift type mixing, and the horizontal disposition of a flat panel PBR would require a new method of mass transfer to become feasible. Conventional tubular systems were implemented successfully as horizontal installations in outdoor environments, and while they could potentially provide a solution to the issue of mass transfer, they struggle to provide ideal shading conditions due to both the spacing between the tubular elements and their refractory properties. Consequently, a hybrid configuration of tubular and flat-panel types in the form of a corrugated flat-panel PBR was developed for this project. It allowed a back-and-forth flowing motion of the culture medium within the panels to be created, resulting in an even distribution of biomass. This type of PBR configuration coupled with the transmittance properties of S. Obliquus offers a great range of operational capabilities as the biomass density within the PBR panels can be altered in response to the varying environmental conditions. Furthermore, this species was already investigated and experimentally characterized in earlier studies from the authors [4,5], and its light transmittance as a function of the biomass density had already been modeled through daylighting simulations.

- Sustainable design strategy: a strategy was developed to improve aspects of sustainability and resource management, with the aim to benefit not only The Edge, but also the broader context of the cultural precinct. Two essential resources were considered, water and electricity, as both are used in the operation of the PBR system as well as in the workspace below it:

- Water strategy: this was proposed to exploit the current potential of the cultural district for rainwater harvesting, as well as the capacity of the microalgae for wastewater treatment. The large roof area of the state library offered a potential catchment of over $6000 \mathrm{~m}^{2}$ for the collection of rainwater, to be utilized both in the library facilities and in the PBR system. Along with the harvested rainwater, greywater derived from library facilities was also to be used in the PBR system where a process of bio-treatment would render the outflow of water suitable for use in irrigation and other potential applications.

- Electricity strategy: photovoltaic cells were to be positioned on the rooftop of the Gallery of Modern Art to offset the energy usage of the PBR system as well the library facilities. In order to reduce the energy demands of the PBR system, a heat transfer system was conceived 

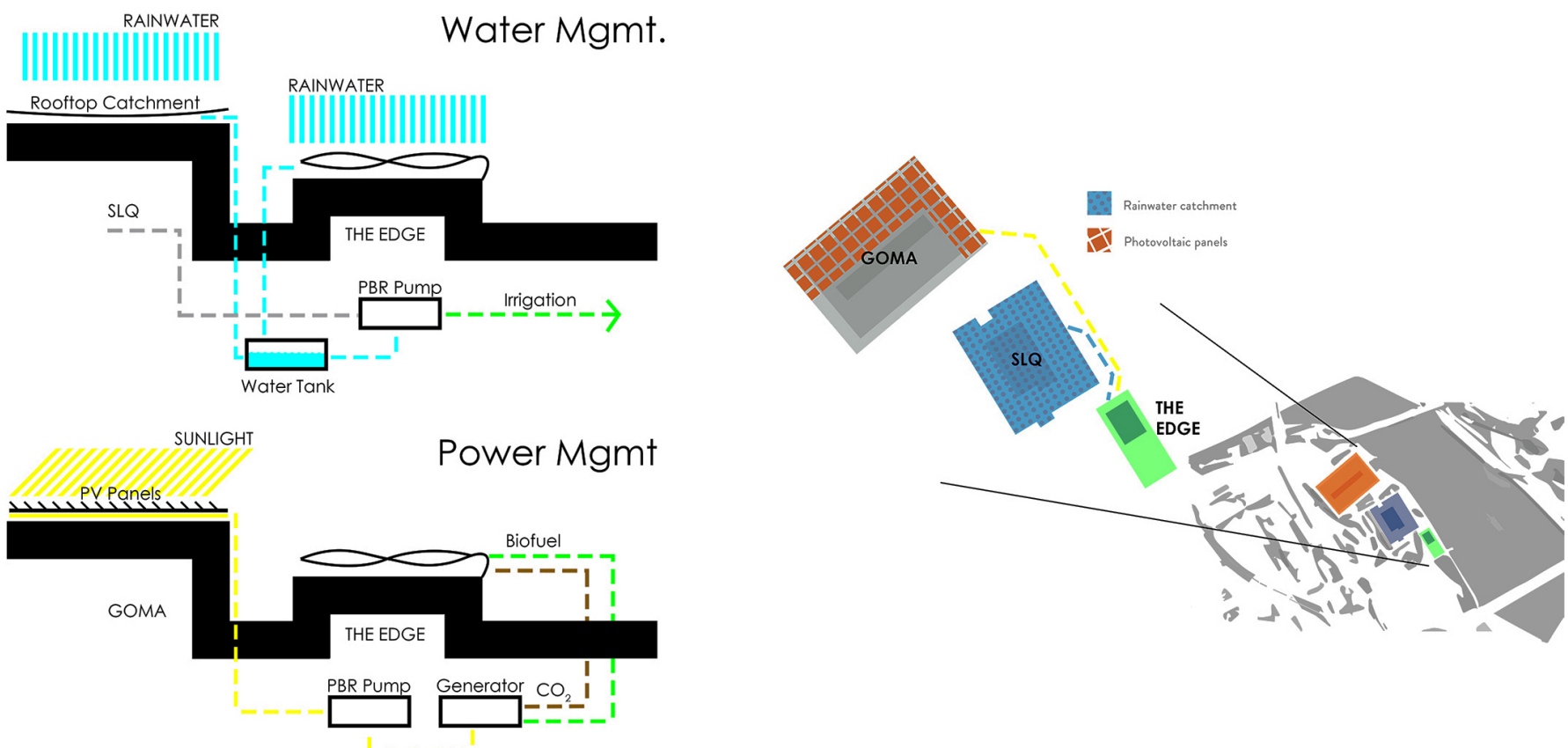

(a)

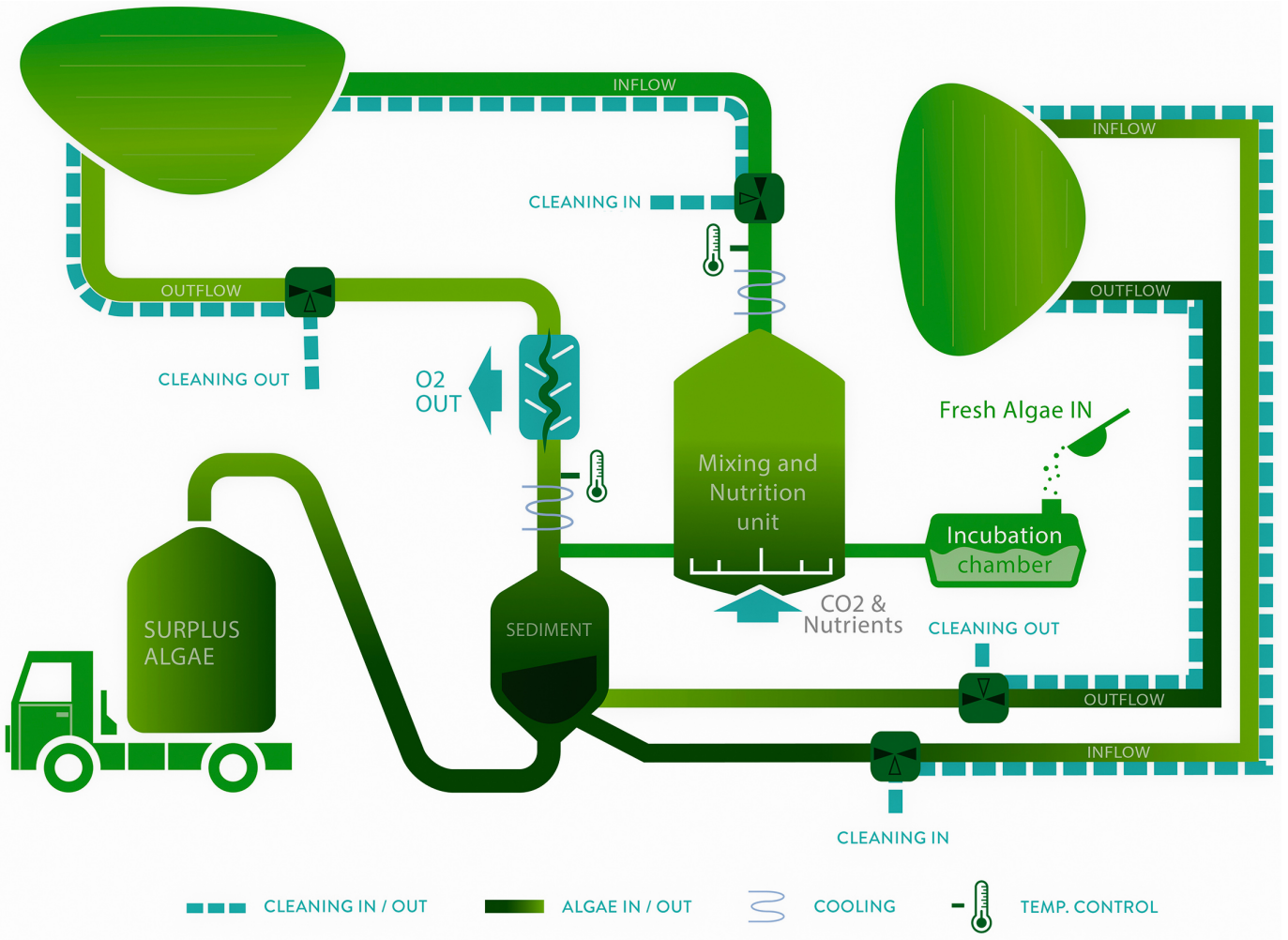

(b)

Fig. 2. Concept of the microalgae cultivation system: schema of (a) the water management strategy and (b) of the power management strategy.

which made use of the readily available cool water from the river to regulate the temperature of the culture medium.

In addition to these strategies, the operational processes of the system were defined to maximize efficiency. In this definition the surplus biomass produced by the PBR system is removed for processing offsite, while the resulting biofuel is then returned to the system to produce electricity and heat. The $\mathrm{CO}_{2}$ produced by the combustion of the biofuel is directed into the PBR system where it is consumed by the microalgae and converted into oxygen. Figure 2 visualizes the concept of the power and energy management strategy, as well as that of the microalgae cultivation system. Finally, it is worth stressing that the sustainability strategy also involves the possibility of reusing and recycling materials: the 
microalgae, at the end of their lifetime as living organisms may be reused in other sectors such as nutraceutics or biodiesel, similarly to a reuse approach already used by the Authors in a previous study [44].

\subsection{Final architectural and technological project}

Various PBR configurations and structural forms were explored through a reiterative process. Among these, a solution with a tubular brise-soleil seemed the most promising as it provided better culture distribution and mass transfer. However, preliminary light simulations showed an uneven distribution of shading due to the spacing and refractory properties of the tubular elements. This issue of homogeneous transmittance was later addressed through the definition of the new PBR elements.

The supporting structure was divided into two parts: the columns, to be cast individually using a system of molds made from CNC machined foam blocks, and the screening element, to be cast as a perforated shell encasing the column tops and consolidating the support structure. In order to accommodate the irregular geometry, the columns were to be cast in a lightweight, structural, self-compacting concrete (SCC). A hollow steel core functioned both as support for the steel reinforcements, and as a means to reduce the overall weight of the elements while allowing for utilities to be concealed within the structure. The perforated shell was to be cast in Ultra High Performance Concrete (UHPC), to minimize the thickness of the shell while providing adequate strength. UHPC is inherently completely weatherproof, thus eliminating the need for additional weatherproofing components.

The final configuration of the PBR shading system was made up of horizontal and vertical panels, whose main components are transparent panes that serve to expose the culture medium to sunlight. UV-filtering, colorless formulations of Plexiglas were selected for this purpose, as they allow for maximum transparency while providing high values of flexural and tensile strength. The UV stability and weatherability of Plexiglas makes it ideal for external use, as opposed to other transparent materials such as polycarbonates, which tend to go yellow or develop appreciable haze levels with prolonged exposure to UV light. Any material used in the PBR elements faced a major obstacle: the direct contact between the transparent surfaces and the culture medium, which
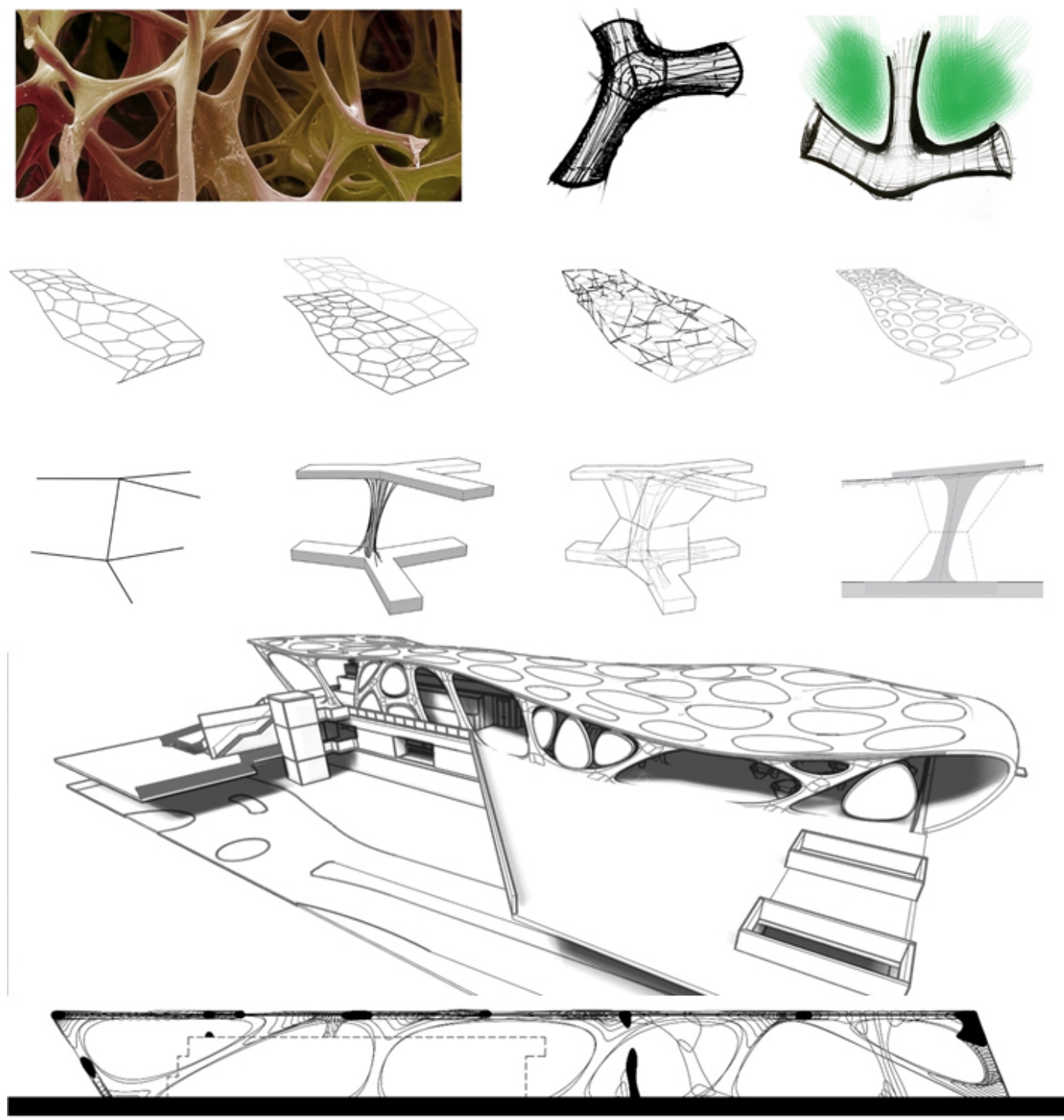

Fig. 3. Parametric evolution of the structure to support the PBRs, from the initial idea (observation of bone tissues) to the final roof geometry. 
makes them subject to a phenomenon known as biofouling. This occurs when microorganisms such as plants, algae, or animals accumulate on a wet surface. In systems that rely on sunlight, biofouling can become a major drawback as it limits the amount of light entering the PBR, which in turn significantly reduces the level of productivity. A number of solutions exist for the issue of biofouling in the form of materials and coatings, the most promising of which were the new super-hydrophobic surface treatments that have been shown to minimize the phenomenon of biofouling, in certain cases eliminating it altogether.

Quite literally, microalgae were at the (living) core of this concept, and the use of organic geometry served as a means to convey this fundamental theme through a physical medium. Historically, architects have looked to nature as a source of inspiration and insight both for aesthetic and structural solutions. Seeing as collaboration with nature is the essential message of this concept, an attempt was made to define the structure through organic forms in reference to this theme of bio-architecture.
A parametric design was carried out using Grasshopper, an addon for Rhino, to create an initial model for the structure and to evolve the process of generating the organic geometry. Taking inspiration from forms found in bone tissue, a concept model was developed in the form of a morphed post-and-lintel structure supporting a horizontal plane of PBR panels. The solid structure was shaped as a sinuous lattice while a membrane of PBR panels seals the upper horizontal plane functioning as the transparent element and filtering the incident daylight. Figure 3 summarizes the evolution of the shape of the structure to host the PBRs, from the initial idea to the final geometry.

The primary function of the horizontal panels was to regulate the amount of daylight transmitted to the workspace; this was accomplished by adjusting the biomass density of the culture medium circulating within the panels. By increasing the biomass density, more daylight is absorbed along the light path resulting in less light exiting the panel, and vice versa. To do so, the circulation of biomass was to be autonomously controlled by monitoring
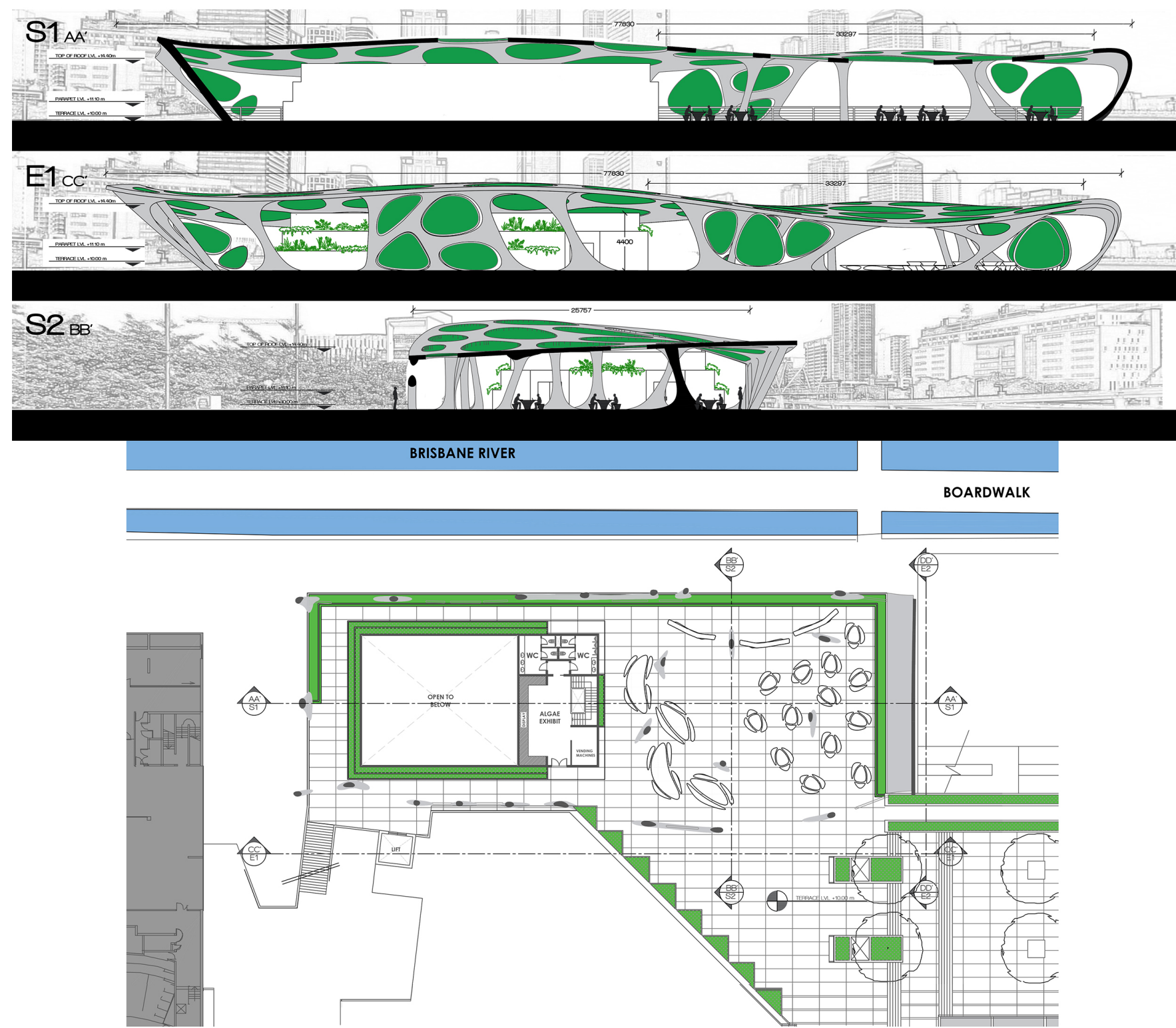

Fig. 4. Plan view, sections, and elevations of the edge's rooftop terrace with the addition of the algae PBRs. 
software, which combines input from an array of photosensors with weather forecast data. This allowed the shading system to adapt to variations in the environmental conditions and facilitate the management of the microalgae culture by estimating biomass yield and forecasting stock culture requirements. The photosensors embedded in the structure measure light levels in two areas, first at the outer surface of the PBR panels and again at the level of the workplane below. It would then use this input to maintain a target level of illumination by regulating the transmittance of the PBR panels along with electric lighting to ensure optimal working conditions.

The design of the PBR panels accounted for fundamental aspects such as flexibility, transparency, responsivity, weatherability, and ease of maintenance. The goal was to simplify their production as much as possible while allowing each panel to have a unique form. The materials used for the transparent component in the PBRs allowed light transmission while at the same time providing sufficient structural strength to support the

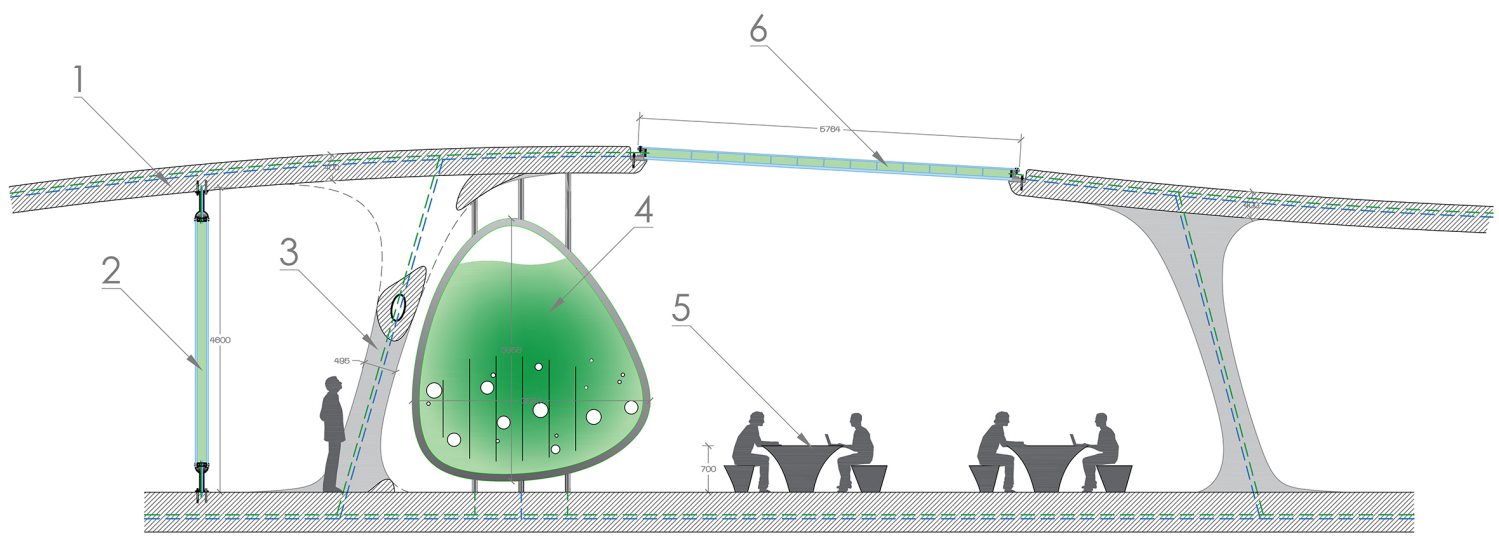

Fig. 5. Representative section of the multi-purpose workspace (caption: 1: concrete shell, 2: vertical panel, section, 3: sectioned column, 4: vertical panel, front view, 5: furnishing, and 6: horizontal panel, section).

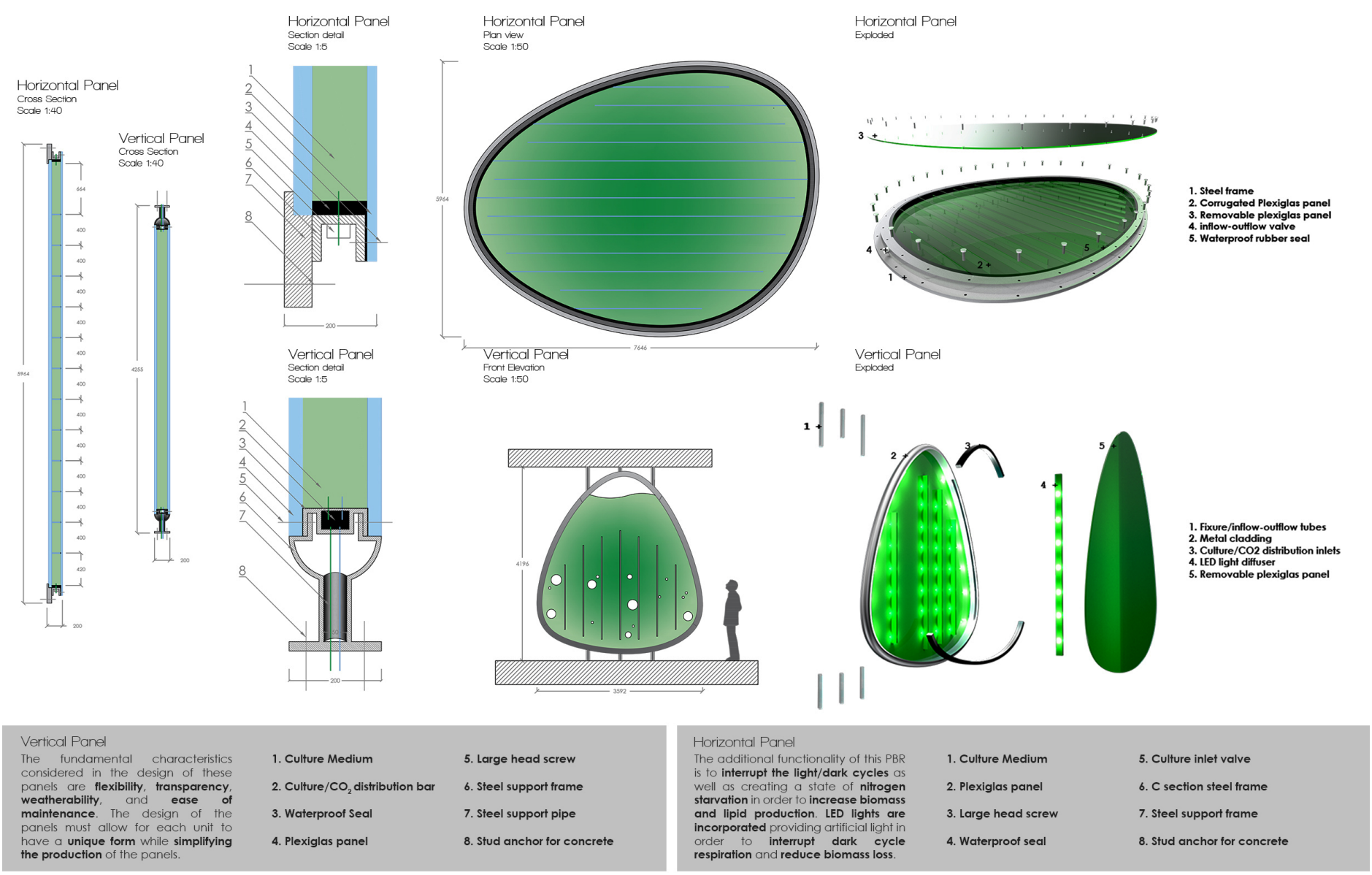

Fig. 6. Details and descriptions of the vertical and horizontal PBR panels (caption: 1: concrete shell, 2: vertical panel, section, 3: sectioned column, 4: vertical panel, front view, 5: furnishing, and 6: horizontal panel, section). 
culture medium. All materials used in the panels were chosen for their ability to withstand exposure to the external elements. Accordingly, a concept for the panels was developed which incorporated a rolled steel frame as the supporting structure and Plexiglas panels for the transparent components. The panels were to be bolted to the structural frame facilitating the removal of the upper panel for routine maintenance.

A second type of vertical PBR was added to the design to enhance the efficiency of the system. Their additional functionality was to create a state of nitrogen deficiency to stimulate an increase in lipid production [45]. Additionally, LED lights were incorporated into the PBR providing electric light in order to interrupt dark cycle respiration, reducing biomass loss. The lighting from these vertical panels also plays a part in the previously mentioned urban lighting display, to be considered as part of the creative lighting strategy.

The interior space of the pavilion was repurposed to double as an exhibition space and restoration point, displaying the project's PBR technologies and sustainable design strategies. Within the exhibition space, functioning PBR elements were to be arranged alongside informative displays that provide a comprehensive demonstration of the technologies and sustainable design strategies of the project. The addition of an exhibition space served to showcase the PBR technology used in the project and educate visitors on the advancement and potentials of this technology for the future of sustainable building.

Figures 4-7 show the final definition of the project.

\section{Method: daylighting simulations}

The daylighting conditions in the workspace on the rooftop terrace area were studied through simulations be means of the daylighting and energy modeling software DIVA-for-Rhino 3D.

For daylighting analyses, this tool uses the validated Radiance algorithm and relies on the daylight coefficient method and the Perez All-Weather Sky model to run annual calculation of daylighting quantities (illuminances and luminances) and to render images of the space under examination. DIVA uses a climate file of the site considered, and therefore it runs annual simulations with a typical time-step of one hour. Besides, it is possible to run simulations for specific time-steps during the course of a year, such as the solstice and equinox days. Taking advantage of this, different sets of simulations were run to obtain the following information:

- illuminance values for specific time-steps during a year: for this analysis, point in time (PIT) illuminance were calculated for June $21^{\text {st }}$, September $21^{\text {st }}$, and December $21^{\text {st }}$, at 9 in the morning and 15 in the afternoon; furthermore, two opposite sky conditions were considered: sunny sky with sun and overcast sky. For each condition, simulations were repeated using varying levels of transparency for the PBR panels. The goal of this set of simulations was to understand the performance of the PBRs under extreme opposite daylight conditions, to determine the various levels of biomass density needed (and therefore light transmittance $\mathrm{T}_{\mathrm{v}}$ ) to still guarantee acceptable illuminance levels on the terrace area under dynamic conditions. In this regard, five $\mathrm{T}_{\mathrm{v}}$ were selected for the analyses: $10 \%, 20 \%, 40 \%, 60 \%, 80 \%$. The connection between biomass density and $\mathrm{T}_{\mathrm{v}}$ and the consequent modeling of the various $T_{v}$ in DIVA were carried out based on the experimental campaigns described in an earlier study by the authors [4].

- aggregate annual daylighting performance: for this analysis, synthetic climate based daylighting metrics CBDM were used, such as the Daylight Autonomy DA [46] and the Useful Daylight Illuminance UDI $[47,48]$. These concepts, defined for indoor daylighting, were adapted to outdoor daylighting. Particularly, different illuminance levels were chosen as threshold to investigate comfort conditions across the terrace area:

o illuminance threshold of $300 \mathrm{~lx}$, which is the minimum illuminance to be guarantee on workplane of an indoor reading area, according to the European standard EN 12464-1:2011 [49]; such threshold was therefore assumed as a lower threshold, under which illuminance should not drop when the $T_{v}$ of the PBRs is changed in response to the external daylight availability

o illuminance threshold of $3000 \mathrm{~lx}$, which is the upper threshold (again, for an indoor space) set by the inventors of the UDI metrics over which a potential glare may be detected $[47,48]$; this was assumed as upper threshold that illuminance in the outdoor workspace should not exceed to prevent from potential glare

○ illuminance threshold of $6000 \mathrm{~lx}$, which was assumed as a secondary upper threshold over which a discomfort glare may be perceived by the users of the terrace, less strict than the upper threshold of 3000 lx.

The goal of this phase was to calculate the daylight performance of the PBRs on an annual basis, with different light transmittance $\mathrm{T}_{\mathrm{v}}$. For this reason, the following two metrics were calculated, relative to the above threshold:

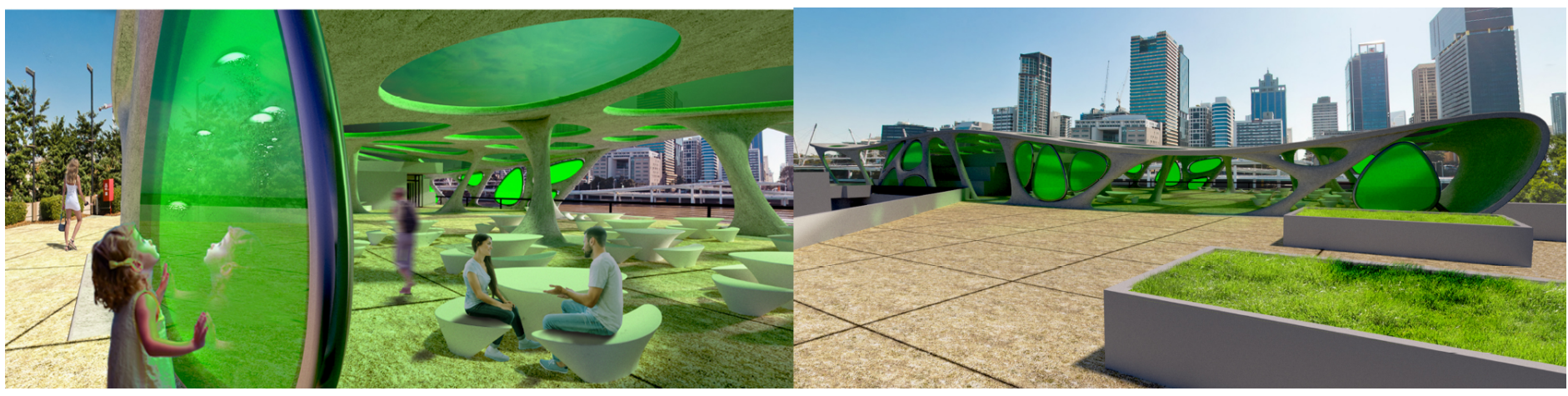

Fig. 7. Renderings of the rooftop terrace area with the algae PBR. 
- $\mathrm{DA}_{>3000}$, that is the percent of occupied hours when daylight illuminance exceeds $3000 \mathrm{~lx}$

- $\mathrm{DA}>6000$, that is the percent of occupied hours when daylight illuminance is over $6000 \mathrm{~lx}$.

For visual comfort purposes, the $\mathrm{DA}>3000$ and $\mathrm{DA}>6000$ should be minimized, while their complementary concepts $\mathrm{DA}_{<3000}$ and $\mathrm{DA}_{<6000}$ (percent of occupied hours when illuminance is below $3000 \mathrm{~lx}$ or $6000 \mathrm{~lx}$, that is in the comfort range) should be maximized. The ideal comfort range is therefore 300-3000 lx (optimal range), which can be extended to 300-6000 lx (acceptable range). Accordingly, also the percent of occupied hours when illuminance is in the 300-3000 lx range or in the 300-6000 lx range where calculated, through the $\mathrm{UDI}_{300-3000}$ and $\mathrm{UDI}_{300-6000}$ metrics. Consistently with the definitions of DA and UDI metrics, the $\mathrm{UDI}_{300-3000}$ and $\mathrm{UDI}_{300-6000}$ were calculated as follows: $\mathrm{UDI}_{300-3000}$ $=\mathrm{DA}_{>3000}-\mathrm{DA}_{>300} ; \mathrm{UDI}_{300-6000}=\mathrm{DA}_{>6000}-\mathrm{DA}_{>300}$. It is worth noticing, that strictly speaking referring to the mere definitions of DA and UDI metrics, $\mathrm{DA}_{>6000}$ has the same meaning of $\mathrm{UDI}_{>6000}$, and similarly $\mathrm{DA}_{>3000}=\mathrm{UDI}_{>3000}$ (both expressing the percent of occupied hours during the course of a year when illuminance due to daylight alone is over $3000 \mathrm{~lx}$, or $6000 \mathrm{~lx}$ ).

As for the DIVA simulations, the following Radiance parameters (Radiance being the validated daylighting algorithm implemented in DIVA) were set: ab 5, ad 1000, as 20, ar 300, aa 0.05 . In the absence of a pre-defined occupancy pattern for the multi-purpose workspace on the terrace, annual simulations were run to calculate DA and UDI metrics, using a schedule from 8 'till 18 , each day of the year (this resulting in 3650 occupancy hours/year). Actually, this is the reference schedule assumed for the calculation of the spatial Daylight Autonomy [50,51] and it allows a 'picture' of the daylighting conditions in the space to be done throughout the year under standard conditions.

The PBRs were modeled as trans materials in DIVA-for-Rhino, starting from experimental data measured by the authors in previous studies $[4,5]$ : actually, the transmittance mode through a glazing + algae system is similar to the one through a translucent way, so a trans material appeared the most suitable modeler in Radiance. The PBRs under examination in the study were coupled with a double plane glazing with a light transmittance of $75 \%$. As five different $\mathrm{T}_{\mathrm{v}}$ were meant to be simulated $\left(\mathrm{T}_{\mathrm{v}}=10 \%, 20 \%, 40 \%\right.$, $60 \%, 80 \%$ ), the following packages were modeled:

- double pane glazing $\left(\mathrm{T}_{\mathrm{v}}=0.75\right)+\mathrm{PBR}$ with $\mathrm{T}_{\mathrm{v}}=0.10$ : globally modeled as a trans with diffuse light transmittance $\mathrm{T}_{\mathrm{v} \text {,diffuse }}=$ 0.075 (product of $0.75 \times 0.1$ ), and diffuse light reflectance $\mathrm{T}_{\mathrm{v}, \text { diffuse }}=0.20$.

- double pane glazing $\left(T_{v}=0.75\right)+$ PBR with $T_{v}=0.20$ : globally modeled as a trans with diffuse light transmittance $\mathrm{T}_{\mathrm{v}, \text { diffuse }}=$ 0.15 (product of $0.75 \times 0.2$ ), and diffuse light reflectance $\mathrm{T}_{\mathrm{v}, \text { diffuse }}=0.15$.

- double pane glazing $\left(T_{v}=0.75\right)+P B R$ with $T_{v}=0.40$ : globally modeled as a trans with diffuse light transmittance $\mathrm{T}_{\mathrm{v} \text {,diffuse }}=$ 0.30 (product of $0.75 \times 0.4$ ), and diffuse light reflectance $\mathrm{T}_{\mathrm{v}, \text { diffuse }}=0.10$.

- double pane glazing $\left(\mathrm{T}_{\mathrm{v}}=0.75\right)+$ PBR with $\mathrm{T}_{\mathrm{v}}=0.60$ : globally modeled as a trans with diffuse light transmittance $\mathrm{T}_{\mathrm{v} \text {,diffuse }}=$ 0.45 (product of $0.75 \times 0.6$ ), and diffuse light reflectance $\mathrm{T}_{\mathrm{v}, \text { diffuse }}=0.05$.

- double pane glazing $\left(\mathrm{T}_{\mathrm{v}}=0.75\right)+\mathrm{PBR}$ with $\mathrm{T}_{\mathrm{v}}=0.80$ : globally modeled as a trans with diffuse light transmittance $\mathrm{T}_{\mathrm{v} \text {,diffuse }}=$ 0.60 (product of $0.75 \times 0.8$ ), and diffuse light reflectance $\mathrm{T}_{\mathrm{v}, \text { diffuse }}=0.025$.

With regard to the site, the simulations were run in two steps:

1. initially for Brisbane (latitude: $27.5^{\circ} \mathrm{S}$, longitude: $153.0^{\circ} \mathrm{E}$ ), which is the town where the building of the case study is located;

2. simulations were then repeated for two other sites: Turin, Italy (latitude: $45.2^{\circ} \mathrm{N}$, longitude $7.6^{\circ} \mathrm{E}$ ) and Dubai, United Arab Emirates (latitude: $25.1^{\circ} \mathrm{N}$, longitude: $55.2^{\circ} \mathrm{E}$ ). This was done to understand how a different setting in terms of biomass density (and thus $T_{v}$ of the PBRs) may be needed for different sites.

These three sites are quite different in terms of daylight availability and solar exposure, both in absolute terms (global illuminance) and in terms of a different ratio of the direct to diffuse components. To quantify this, the luminous exposure LE was calculated for the three sites. The LE is a climate parameter, which was introduced in the recent standard EN 15193-1:2017 [52] to

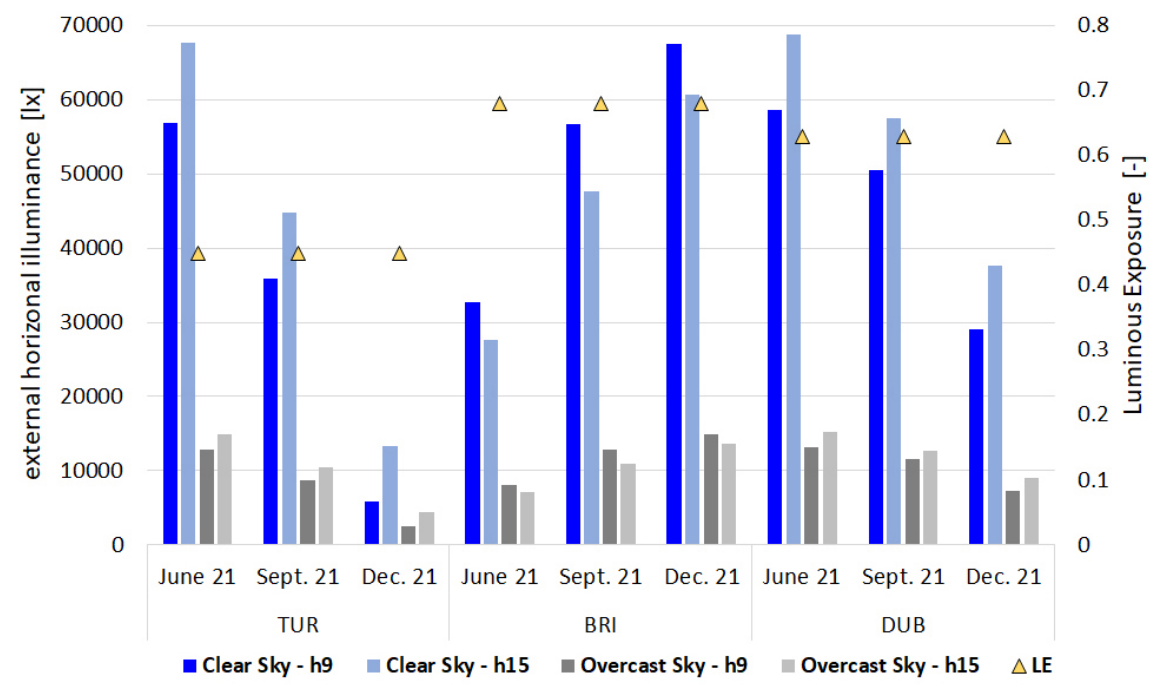

Fig. 8. PIT horizontal illuminance values for each site, without the presence of PBR shading elements (unshaded illuminances). 


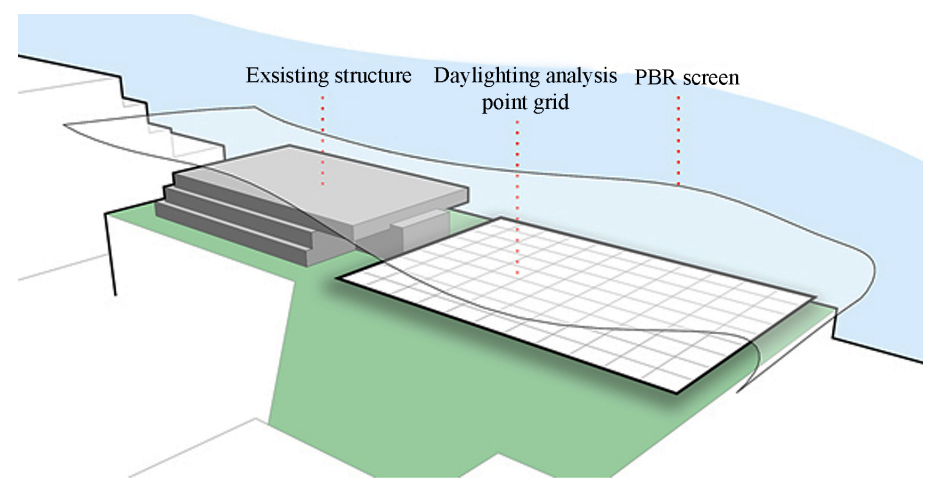

Fig. 9. Schema of the configuration of the site and the positioning of the node grid used for simulations.

synthetically represent the daylight availability of a site, based on its climate. It is defined as the ratio of the direct to the total external horizontal illuminance, both measured or calculated daily from 8 am through $5 \mathrm{pm}$, and then summing up all the daily contributions throughout the 365 days of a year. The climate files of the three locations were used for this calculation and a script in Python was specifically written to extract the LE. The following results were found: for Turin, $\mathrm{LE}=0.45$; for Dubai, $\mathrm{LE}=0.68$, for Brisbane, $\mathrm{LE}$ $=0.63$. Therefore, the locations that were selected represent a quite large variety of climates, ranging from conditions with yearlyround predominant direct sunlight (Dubai and Brisbane, with $\mathrm{LE}=0.68$ and $\mathrm{LE}=0.63$, respectively) to conditions with a quite equal presence of direct sunlight and diffuse skylight (Turin, LE = $0.45)$.

The LE is a synthetic parameter that summarizes the annual solar exposure of a site during the course of a year through one single data. Beside the LE, the specific global horizontal illuminances (in the absence of obstructions) were calculated through DIVA simulations for each site for the different time-steps considered in the study (Fig. 8).

As shown in the Fig. 8, the average illuminance for clear sky conditions is generally higher than $30000 \mathrm{~lx}$ (except for the case of Turin in December), significantly higher than the ideal range of illumination falling between 300 and $3000 \mathrm{~lx}$ (or maximum 6000 $\mathrm{lx}$ ). This demonstrates the need for shading elements to reduce the illuminance levels and create an environment capable of hosting an outdoor workspace. The same applies for overcast skies, though to a lesser extent.

\section{Results}

\subsection{Results for Brisbane}

As a first analysis, the PIT illuminances determined for Brisbane through DIVA simulations are shown in Figs. 9-11.

Figure 9 shows the overall configuration of the site with the existing structure and its relationship to the PBR screen. The area of interest, and object of the daylighting analyses, is the space directly adjacent to the existing structure.

Figure 10 shows a sample of the PIT illuminance data collected for Brisbane, both clear and overcast conditions are represented for the time-step of September 21st at 9 in the morning. Each diagram provides a visual representation (plan view) of the distribution of illuminance values within the target area under a given $\mathrm{T}_{\mathrm{v}}$. The existing structure is represented on the left in grey while each of the adjacent squares corresponds to a single $1 \mathrm{~m} \mathrm{x}$ $1 \mathrm{~m}$ node within the analysis grid. Each set is annotated with the corresponding $\mathrm{T}_{\mathrm{v}}$ along with the mean illuminance values for each set. This initial synthetic analysis indicates the most suitable $T_{v}$ values to be set for the PBRs so as to keep illuminance levels below $3000 \mathrm{~lx}\left(\mathrm{~T}_{\mathrm{v}}=10 \%\right.$ for a clear sky, $\mathrm{T}_{\mathrm{v}}=40 \%$ for an overcast sky), or below the less strict criterion of $6000 \mathrm{~lx}\left(\mathrm{~T}_{\mathrm{v}}=30 \%\right.$ for a clear sky, $\mathrm{T}_{\mathrm{v}}=80 \%$ for an overcast sky).

Complementary, Fig. 11 shows the average illuminance that was determined for all $\mathrm{T}_{\mathrm{v}}$ values $\left(\mathrm{T}_{\mathrm{v}}=10 \%, 20 \%, 40 \%\right.$, and $\left.80 \%\right)$ for all the time-steps considered for the PIT illuminance analysis. The following trends can be highlighted:

- in June (winter period in Brisbane), in the morning (h.9), in the presence of a clear sky the biomass density needs to be set to yield the minimum $\mathrm{T}_{\mathrm{v}}$ of $10 \%$ to guarantee the comfort range

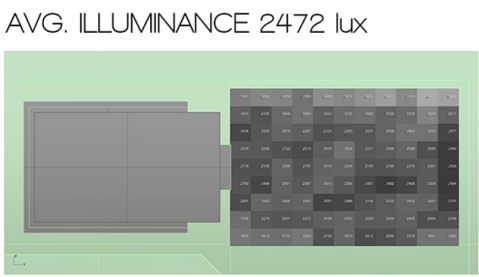

(a)

AVG. ILLUMINANCE 893 lux

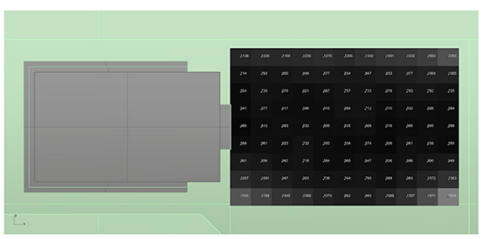

(d)
AVG. ILLUMINANCE 3879 lux

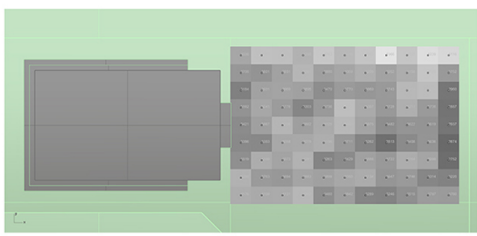

(b)

AVG. ILLUMINANCE 2499 lux

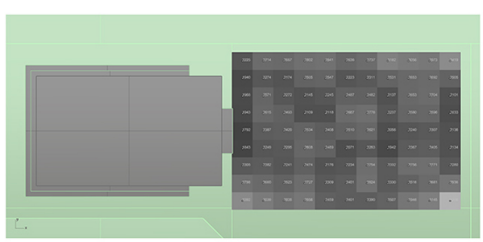

(e)

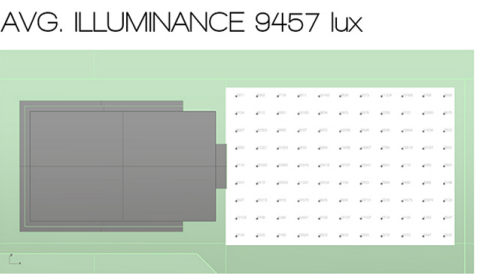

(c)

AVG. ILLUMINANCE 4422 lux

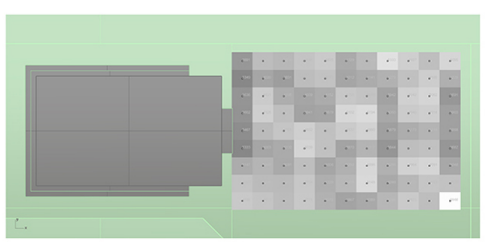

(f)

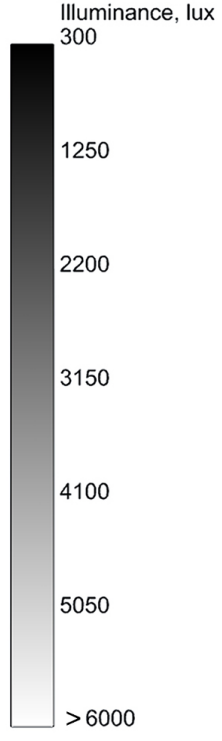

Fig. 10. PIT illuminance values under the PBR shading system for different $T v$ values. Brisbane, on September 21 at 9 in the morning: $(\mathbf{a})$ clear sky and $T_{v}=10 \%$, (b) clear sky and $\mathrm{T}_{\mathrm{v}}=20 \%,\left(\right.$ c) clear sky and $\mathrm{T}_{\mathrm{v}}=40 \%,\left(\right.$ d) overcast sky and $\mathrm{T}_{\mathrm{v}}=10 \%,\left(\right.$ e) overcast sky and $\mathrm{T}_{\mathrm{v}}=20 \%$, and (f) overcast sky and $\mathrm{T}_{\mathrm{v}}=40 \%$. 


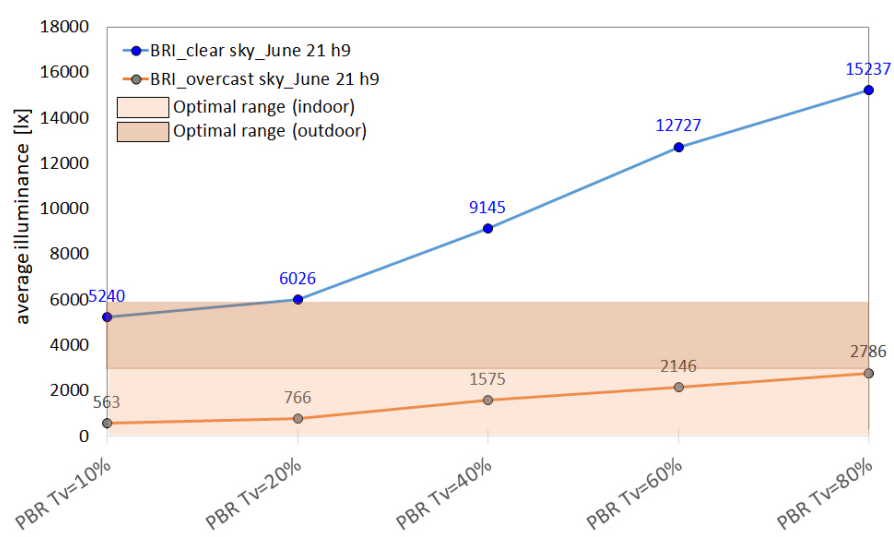

(a)

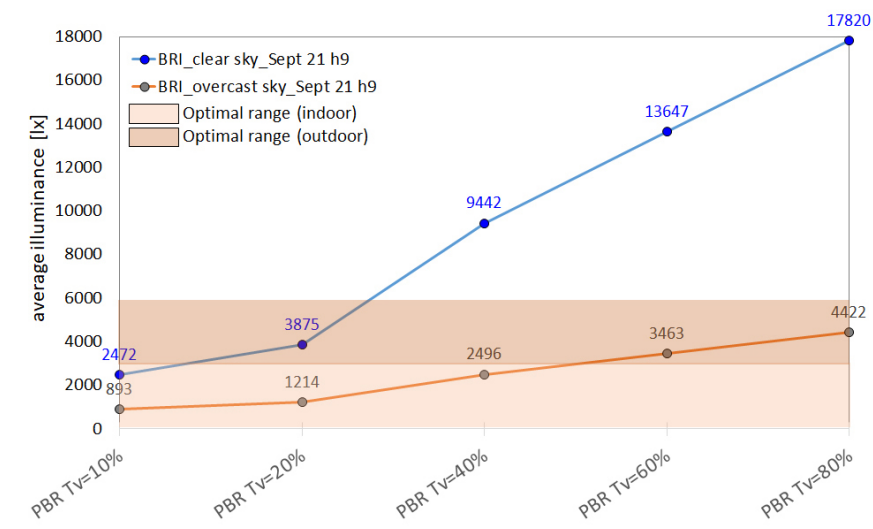

(c)

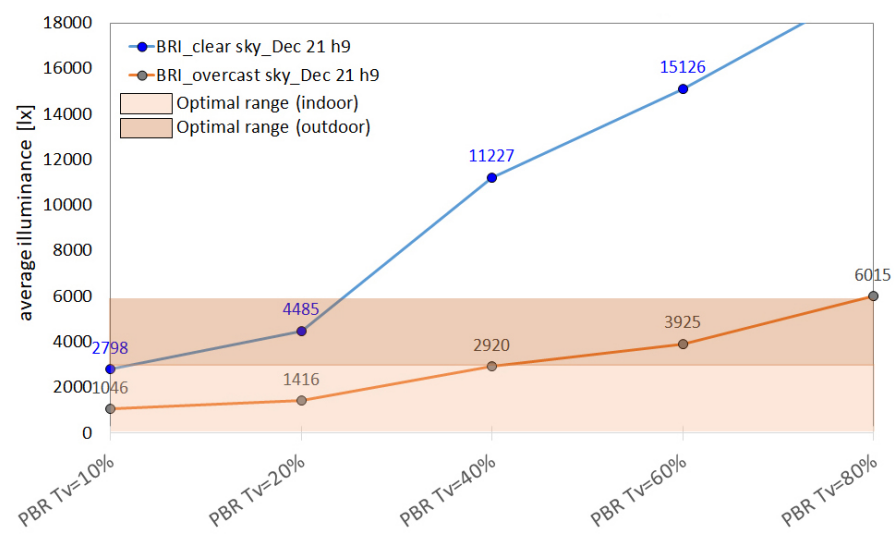

(e)

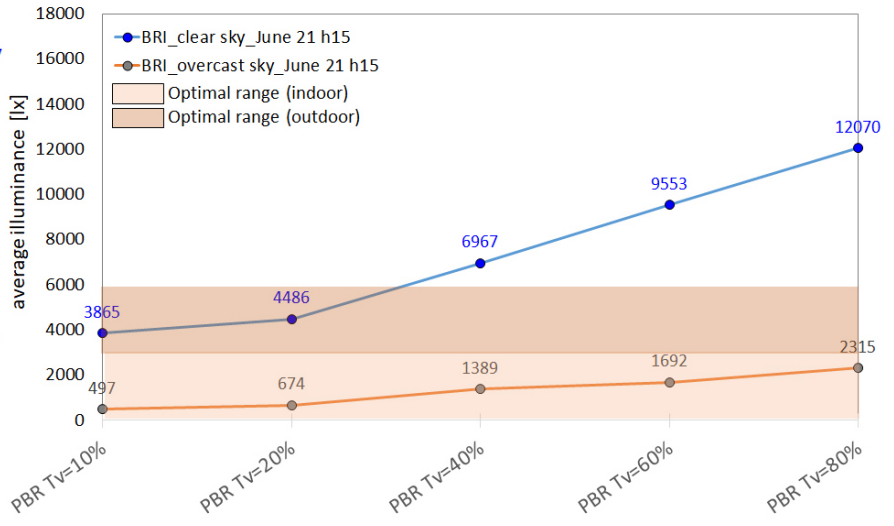

(b)

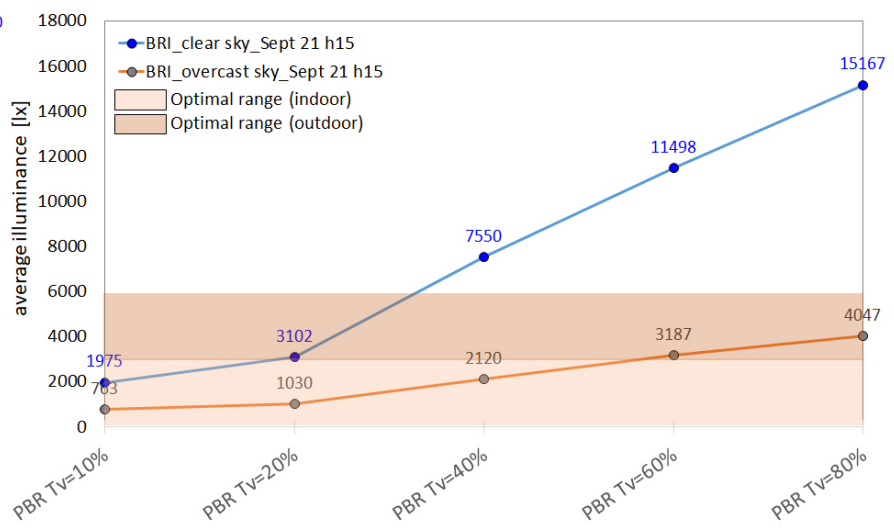

(d)

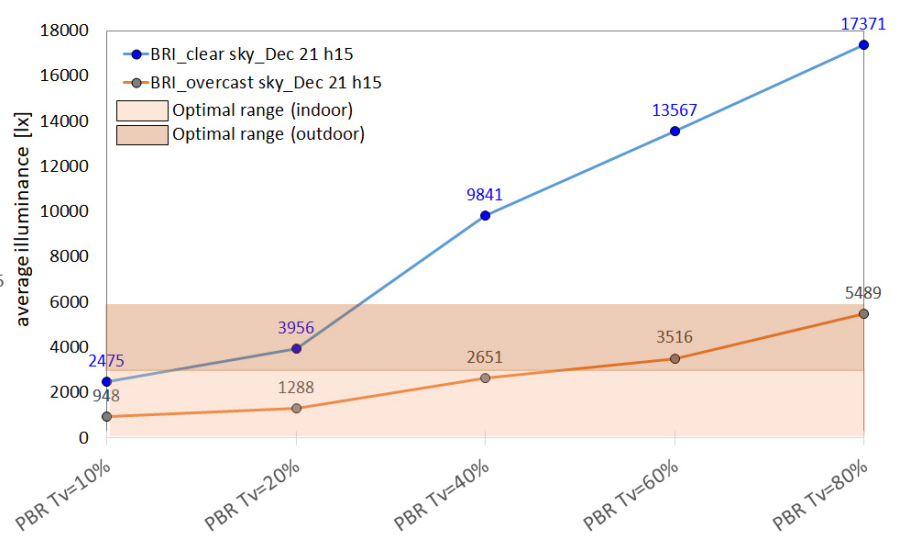

(f)

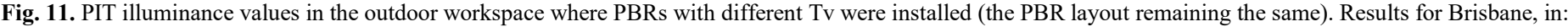
June at (a) h9 and (b) h15, September at (c) h9 and (d) h15, and December at (e) h9 (f) h15.

300-6000 1x, whilst it is not possible to guarantee the comfort range 300-3000 $1 \mathrm{x}$. This comfort range is guaranteed in the presence of an overcast also setting the $\mathrm{T}_{\mathrm{V}}$ of algae to the highest value of $80 \%$. In the afternoon (h.15), the same trend was observed in the presence of an overcast sky, while with a clear sky, the $\mathrm{T}_{\mathrm{v}}$ of PBRs can be set to $30 \%$ to guarantee the comfort range 300-3000 $1 \mathrm{x}$

- in September, in the morning (h.9), in the presence of a clear sky a biomass density that yields the minimum $\mathrm{T}_{\mathrm{v}}$ of $10 \%$ guarantees the comfort range 300-3000 lx, while a value of slightly lower than $30 \%$ guarantees the comfort range 300$6000 \mathrm{~lx}$. In the presence of an overcast, the $\mathrm{T}_{\mathrm{v}}$ of algae needs to be set to $50 \%$ for the comfort range $300-3000 \mathrm{~lx}$ and to $80 \%$ for the comfort range 300-6000 lx. The same settings also apply in the afternoon (h.15)

- in December (summer period in Brisbane), in the morning (h.9), in the presence of a clear sky $\mathrm{T}_{\mathrm{v}}$ values of PBRs of $10 \%$ and $20 \%$ are needed to guarantee the comfort ranges 300-3000 lx and 300-6000 lx, respectively. Under an overcast sky, the Tv of PBRs needs to be set to $40 \%$ for the comfort range 300-3000 $\mathrm{lx}$ and $80 \%$ for the comfort range 300-6000 $1 \mathrm{x}$. The same settings were also observed to be valid for in the afternoon (h.15). 


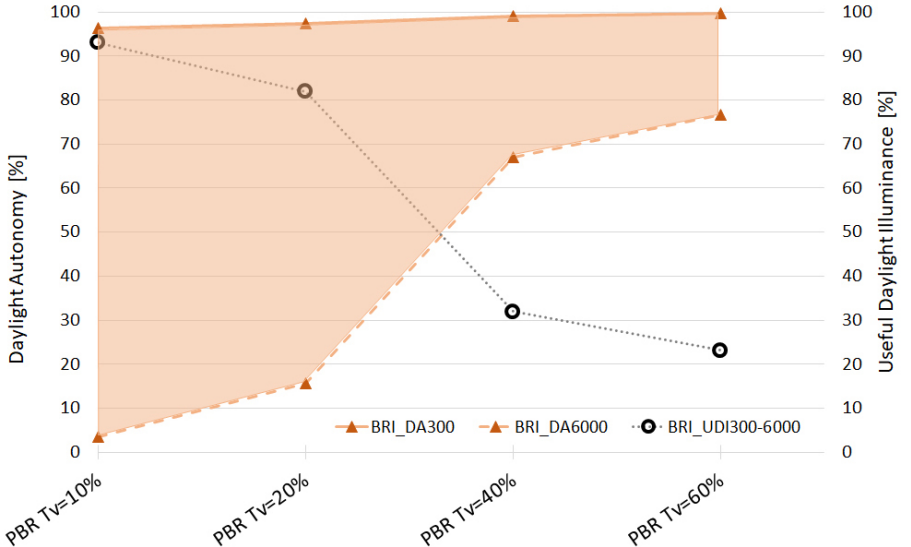

Fig. 12. Annual DA average values in the rooftop terrace area where PBRs with different $T_{v}$ were installed, in Brisbane.

As far as the annual analyses for Brisbane are concerned, Fig. 12 shows the Daylight Autonomy and the Useful Daylight Illuminances that were found from simulations for the various $T_{v}$ of the PBRs considered. As mentioned earlier, optimizing the $\mathrm{UDI}_{300-6000} \mathrm{~lx}$ is the 'acceptable' goal to pursue as one could expect, this is achieved when the minimum $\mathrm{T}_{\mathrm{v}}$ of $10 \%$ is set throughout the year $\left(\mathrm{UDI}_{300-6000}>90 \%\right)$, but a satisfactory performance of $\mathrm{UDI}_{300-6000}>50 \%$ is guaranteed by setting the $\mathrm{T}_{\mathrm{v}}$ of the PBRs to $30 \%$. It is worth stressing that this analysis refers to an hypothetical condition for which the PBRs are kept to the same setting during the full course of a year, which is not the case of the system developed to shade the workspace, whose light transmittance is varied in response to boundary conditions.
Accordingly, this synthetic, annual representation needs to be analyzed in synergy with the analysis of the PIT illuminance shown earlier.

Table 1 summarizes the maximum $T_{v}$ values to which PBRs need to be set for each time-step considered to guarantee the 'optimal' comfort range 300-3000 lx and the 'acceptable' comfort range 300-6000 lx. In short, the following main trends can be outlined:

- in the presence of a clear sky condition (with an external, unobstructed horizontal illuminance in the range 27000-68000 $1 \mathrm{x}$ ), the maximum $\mathrm{T}_{\mathrm{V}}$ of PBRs results is in the range $5 \%-20 \%$ to remain in the 'optimal' comfort range, and in the range $20 \%$ $30 \%$ to remain in the 'acceptable' comfort range;

- in the presence of a clear sky condition (with an external, unobstructed horizontal illuminance in the range 8000-15000 $1 \mathrm{x}$ ), the maximum $\mathrm{T}_{\mathrm{v}}$ of PBRs results in the range $40 \%-80 \%$ to remain in the 'optimal' comfort range, and can be set to the maximum value of $80 \%$ to remain in the 'acceptable' comfort range.

\subsection{Comparison between Brisbane, Turin and Dubai}

Figure 13 expands the earlier analysis, showing the PIT illuminance for all the simulated time-steps (June, September, and December, at 9 and at 15), sky conditions (clear and overcast), and sites considered in this study.

In general, results confirm that in clear sky conditions the target illuminance range can be maintained with moderate levels of biomass saturation $\left(\mathrm{T}_{\mathrm{V}}<20 \%\right.$ for all the sites considered to remain within the 300-6000 lx illuminance range), however optimal results are only seen with high concentrations of biomass density

Table 1. Maximum light transmittance Tv to which the PBRs need to be set under clear and overcast skies for the different time-steps considered in the study.

\begin{tabular}{|c|c|c|c|c|c|c|}
\hline \multirow[t]{2}{*}{ Date } & \multicolumn{3}{|l|}{ Clear sky } & \multicolumn{3}{|l|}{ Overcast sky } \\
\hline & $\mathbf{E}_{\text {ext,horizontal }}[\mathbf{l x}]$ & $\begin{array}{l}\text { Optimal range } \\
300-3000 \mathrm{~lx}\end{array}$ & $\begin{array}{l}\text { Acceptable range } \\
300-6000 \mathrm{~lx}\end{array}$ & $\mathbf{E}_{\text {ext,horizontal }}[\mathbf{l x}]$ & $\begin{array}{l}\text { Optimal range } \\
300-3000 \mathrm{~lx}\end{array}$ & $\begin{array}{l}\text { Acceptable range } \\
300-6000 \text { lx }\end{array}$ \\
\hline June 21 h9 & 32757 & $\mathrm{~T}_{\mathrm{v}}<10 \%$ & $\mathrm{~T}_{\mathrm{v}}<20 \%$ & 8055 & any $\mathrm{T}_{\mathrm{v}}$ & any $\mathrm{T}_{\mathrm{v}}$ \\
\hline June 21 h15 & 27566 & $\mathrm{~T}_{\mathrm{v}}<5 \%$ & $\mathrm{~T}_{\mathrm{v}}<30 \%$ & 7160 & any $\mathrm{T}_{\mathrm{v}}$ & any $\mathrm{T}_{\mathrm{v}}$ \\
\hline Sept. $21 \mathrm{~h} 9$ & 56654 & $\mathrm{~T}_{\mathrm{v}}<10 \%$ & $\mathrm{~T}_{\mathrm{v}}<30 \%$ & 12800 & $\mathrm{~T}_{\mathrm{v}}<50 \%$ & $\mathrm{~T}_{\mathrm{v}}<80 \%$ \\
\hline Sept. 21 h15 & 47707 & $\mathrm{~T}_{\mathrm{v}}<20 \%$ & $\mathrm{~T}_{\mathrm{v}}<30 \%$ & 10919 & $\mathrm{~T}_{\mathrm{v}}<60 \%$ & any $\mathrm{T}_{\mathrm{v}}$ \\
\hline Dec. 21 h9 & 67572 & $\mathrm{~T}_{\mathrm{v}}<10 \%$ & $\mathrm{~T}_{\mathrm{v}}<25 \%$ & 14946 & $\mathrm{~T}_{\mathrm{v}}<40 \%$ & $\mathrm{~T}_{\mathrm{v}}<80 \%$ \\
\hline Dec. $21 \mathrm{~h} 15$ & 60681 & $\mathrm{~T}_{\mathrm{V}}<10 \%$ & $\mathrm{~T}_{\mathrm{V}}<30 \%$ & 13604 & $\mathrm{~T}_{\mathrm{V}}<40 \%$ & $\mathrm{~T}_{\mathrm{V}}<80 \%$ \\
\hline
\end{tabular}

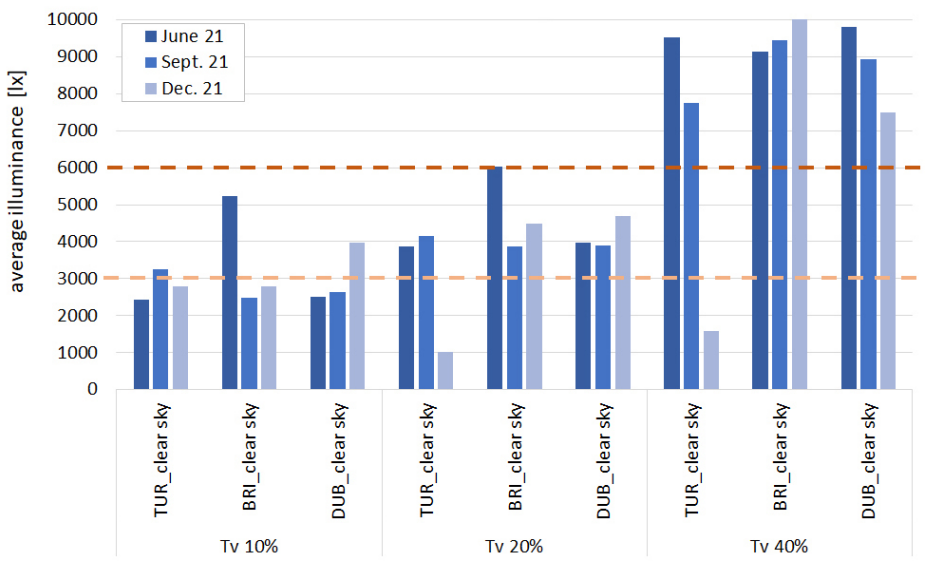

(a)

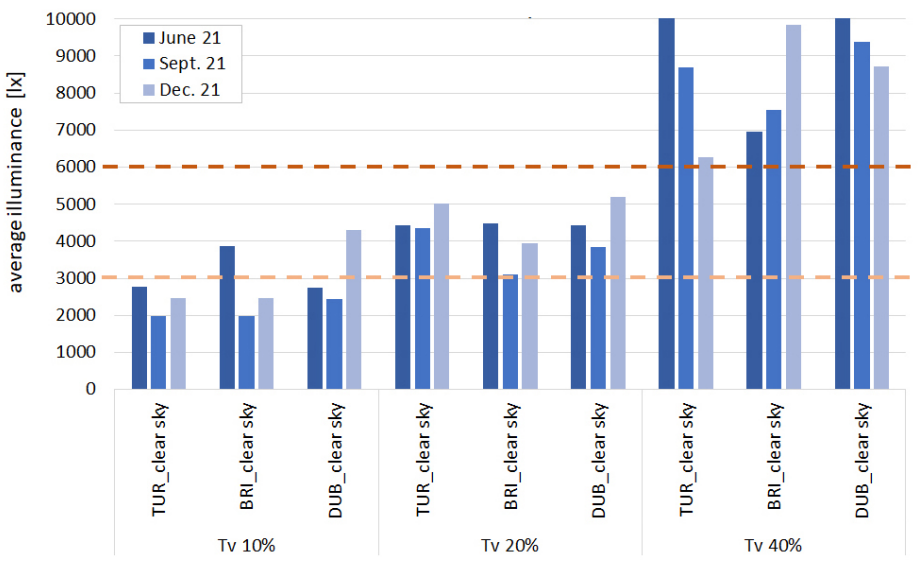

(b) 


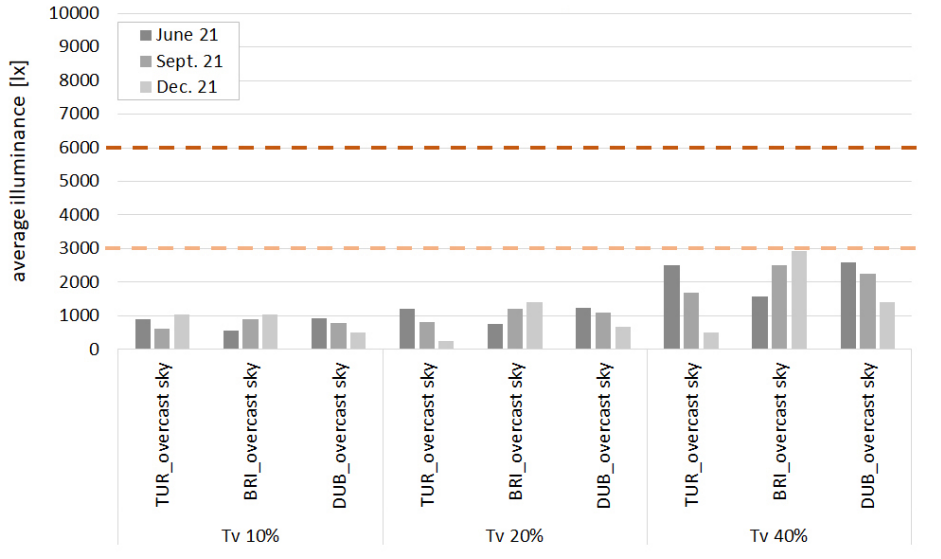

(c)

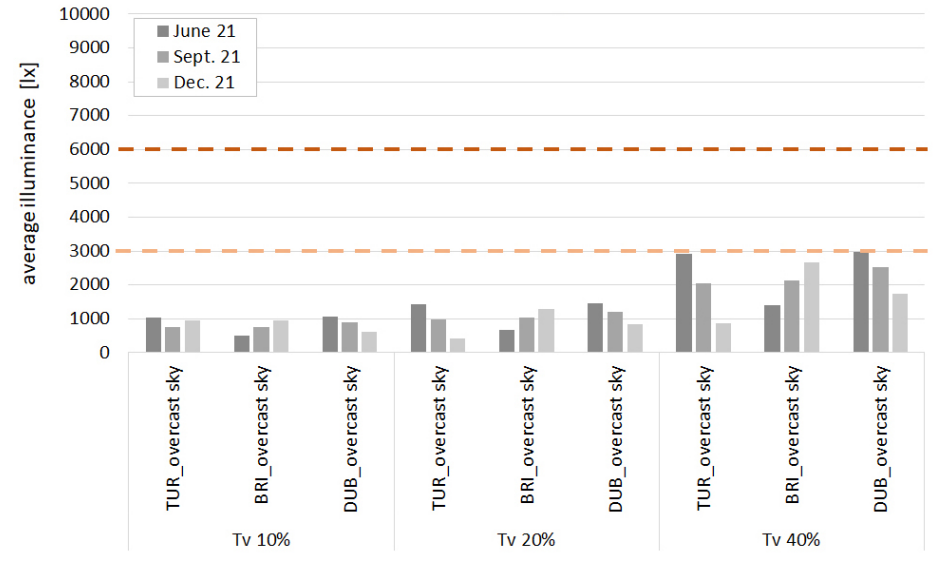

(d)

Fig. 13. Average PIT illuminance values in the rooftop terrace area where PBRs with different Tv were installed (the layout remaining the same). Results for three locations (Brisbane, Turin, Dubai), in different periods of the year and under different sky conditions: (a) clear sky at h9, (b) clear sky at h15, (c) overcast sky at h9, and (d) overcast sky at h15.

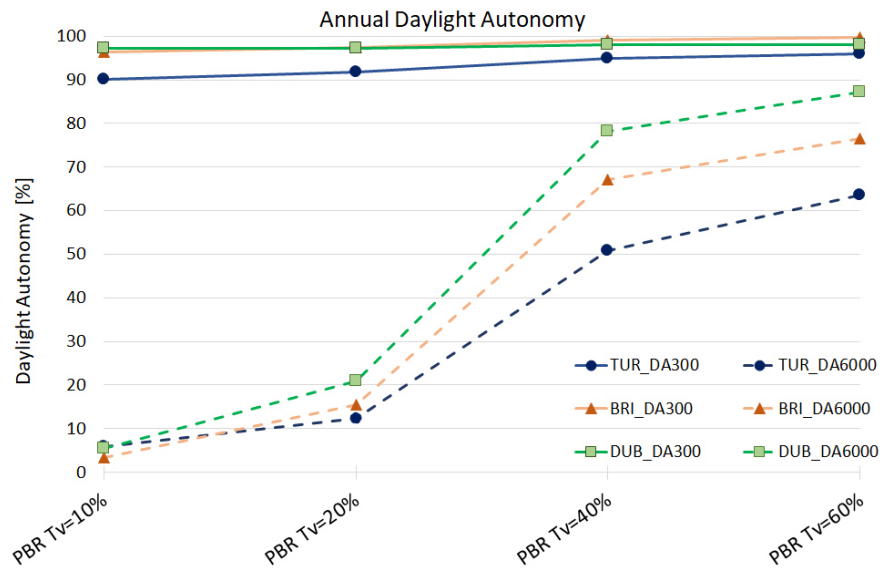

(a)

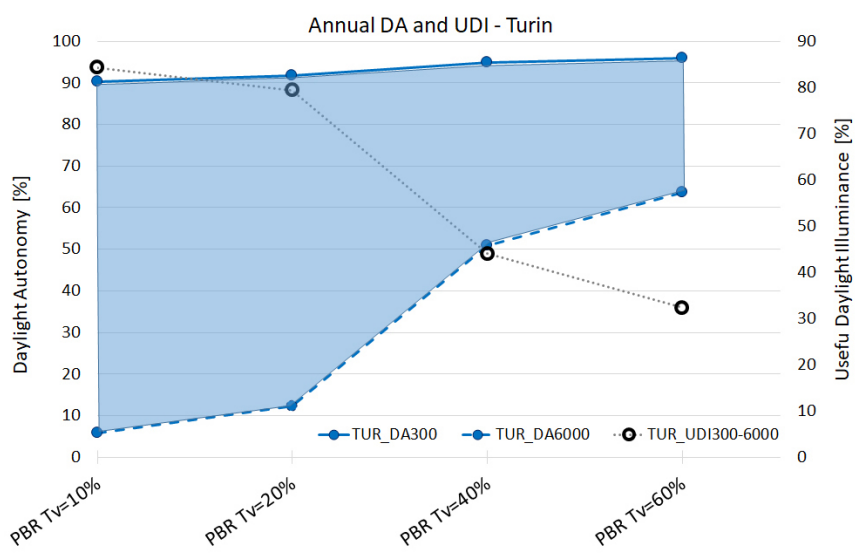

(c)

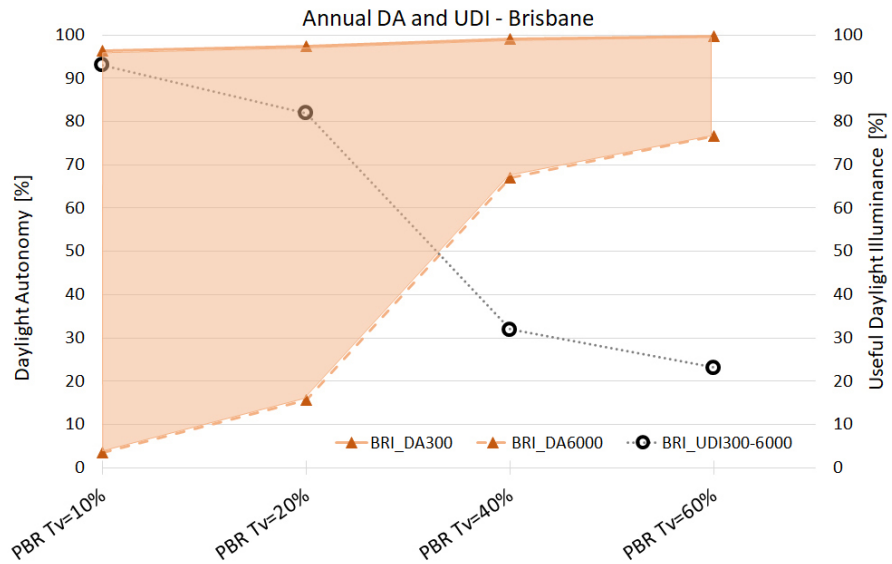

(b)

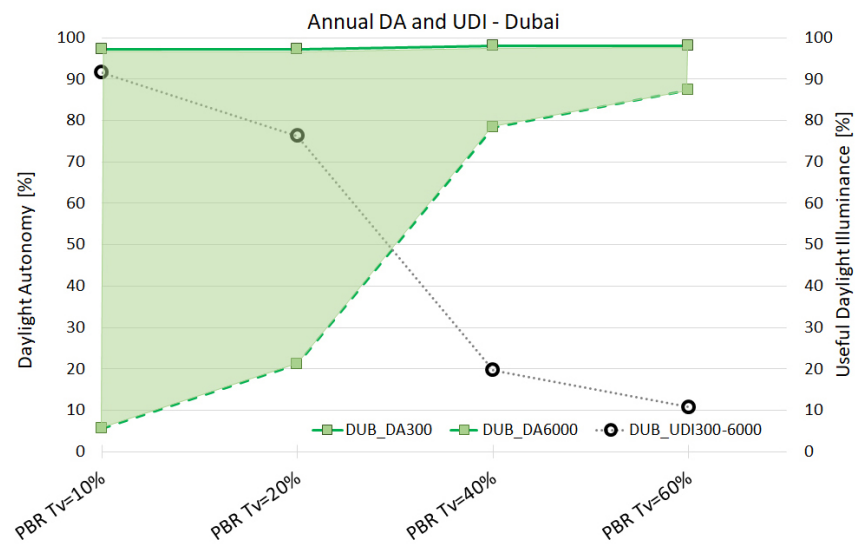

(d)

Fig. 14. Annual DA and UDI average values in the rooftop terrace area where PBRs with different Tv were installed (the layout remaining the same): (a) results for all the three locations, (b) results for Brisbane, (c) results for Turin, and (d) results for Dubai.

$\left(\mathrm{T}_{\mathrm{v}}<10 \%\right.$ for $300-6000 \mathrm{~lx}$ illuminance range). Conversely, in overcast sky conditions the optimal range of illuminance is easily obtained with lower levels of biomass saturation $\left(\mathrm{T}_{\mathrm{v}}<80 \%\right)$.

Annual Daylight Autonomy (DA) data was also collected in support of this study. Figure 14 shows the resulting DA conditions as annual percentages in relation to the various $T_{v}$ values within a
Useful Daylight Illuminance (UDI) threshold of 300-6000 1x. Figure 14(a) compares the values side-by-side, while Figs. 14(b), (c), and (d) focus on individual sites, highlighting the area in which the condition of $\mathrm{UDI}_{300-6000}$ is satisfied.

As shown in Fig. 14(a), the lines for $\mathrm{DA}_{>6000}$ are quite close to each other for the three sites, while for the lower threshold 
$\left(\mathrm{DA}_{>300}\right)$, the line for Dubai highlights higher $\mathrm{DA}_{>6000}$ values compared to the two other sites, especially as the $T_{\mathrm{v}}$ of PBRs increases to values $>40 \%$. Consequently, as one could expect, the largest 'comfort area' (area in the graph that represents $\mathrm{UDI}_{300-6000}$ ) was observed for Turin, while the smallest area was observed for Dubai. This is consistent with the higher presence of direct sunlight, on an annual basis, in Dubai $(\mathrm{LE}=0.68)$ as opposed to Turin $(\mathrm{LE}=0.45)$. The daylighting performance of $\mathrm{UDI}_{300-6000}$ for Brisbane $(\mathrm{LE}=0.63)$ falls predictably between those of Dubai and Turin, and tends to be closer to the results of Dubai.

It is worth stressing that no prescription is given for the DA and UDI metrics for outdoor environments. In principle, the greater the $\mathrm{UDI}_{300-6000}$ values (or, in other words, the lower the $\mathrm{DA}_{>6000}$ values), the better the system is able to provide visual comfort conditions. The graphs highlight through hatches a 'comfort area' (Figs. 14(b), (c), and (d)), that is the distance between the two lines in each graph: this graphical representation of the $\mathrm{UDI}_{300-6000}$ provides an overview of the expected performance of the PBR screening system, which serves as a tool to correlate the biomass density of the PBRs (as a function of $\mathrm{T}_{\mathrm{v}}$ ) with the daylight performance in terms of $\mathrm{UDI}_{300-6000}$ (to be maximized) or $\mathrm{DA}_{<6000}$ (to be minimized). As an example, if the $\mathrm{DA}_{>6000}$ is wanted to remain under $20 \%$ (i.e. illuminance values $>6000 \mathrm{~lx}$ for $20 \%$ of occupied hours of the terrace during the course of a year), this implies to set $\mathrm{T}_{\mathrm{v}}<25 \%$ for Turin, $\mathrm{T}_{\mathrm{v}}<22 \%$ for Brisbane, and $\mathrm{T}_{\mathrm{v}}$ $<20 \%$ for Dubai. Alternatively, in order to achieve $\mathrm{DA}_{>6000}<40 \%$ (i.e. illuminance values $>6000 \mathrm{~lx}$ for $40 \%$ of occupied hours on an annual basis), $T_{v}$ needs to set to the following values for the the different sites: $\mathrm{T}_{\mathrm{V}}<34 \%$ for Turin, $\mathrm{T}_{\mathrm{v}}<29 \%$ for Brisbane, and $\mathrm{T}_{\mathrm{v}}$ $<27 \%$ for Dubai.

\section{Discussion}

\subsection{Sustainability issues}

Microalgae cultivation as a renewable resource has been the object of great interest in recent years; the scientific and industrial communities are continuously developing new concepts to take advantage of the ample research that has been produced over the past decade. This study proposed a diverse application allowing this predominantly industrial technology to find new opportunities within cities. The study is aligned with the theory behind numerous European urban redevelopment projects (Concerto Projects) which have used the production of energy on a widespread local scale as a driver for the district's urban renovation [53]. In addition to its inherent benefits, the cultivation of microalgae in urban settings may also allow for the creation of unique spaces capable of offering new ways of interacting with the built environment. Sustainability and environmental responsiveness are among the primary themes of architectural discourse and this study aimed to take advantage of PBRs as a means to better satisfy these primary goals, and to underline the relevance of this technology as an element in sustainable construction. The applications of the proposed system is potentially very broad: following the most widespread technologies of solar energy capture (photovoltaic and solar thermal), the algae PBR system can exploit the glass surfaces of large urban buildings. Moreover, thanks to the aesthetic and formal potential, this technology can in fact be adapted to the building envelope very easily by varying its shape, size and structure; the panels do not inherently have a defined shape, giving them the ability to be adapted to the architectural language of the building in consideration.

The lighting simulations created for this study made use of practical data gathered from previous investigations into the behavior of PBRs and the results showed promise for the application of PBRs as dynamic shading elements. The research carried out in support of the development of a sustainable design strategy also showed the potential of this technology not only as a source for a wide variety of valuable resources and a means for bio-sequestration of $\mathrm{CO}_{2}$, but also for providing additional benefits to environmental quality and wellbeing.

The lack of practical data, one of the driving forces behind the development of the BIQ house in Hamburg, means that many of the real-world issues that will be faced in the realization of such a project cannot be fully quantified. Issues such as operational costs, environmental conditions, harvesting efficiency, illumination and visual comfort, are only a few examples of the parameters that needed to be approximated in this study. Considerations must be made to allow for a degree of experimentation and testing as an integral part of its successful execution, and the correct implementation of microalgae cultivation technology in the early stages will undoubtedly have a lasting influence on its potential for future development. It is therefore imperative to address, as far as possible, all socio-economic and environmental issues relevant to the pursuit of practical solutions; as in the case of this study such a solution may present itself in the form a synthesis between complimentary renewable resources, in which the weaknesses of the individual systems are offset by their combined effect.

Education, awareness, and information are a fundamental aspect in the development and implementation of sustainable technologies. As an essential part of this study, an exhibition space is envisioned with the aim of creating a dedicated space for the showcasing of microalgae cultivation technology, as well as an education hub for on the advancements and potentials of all efforts in the creation of a sustainable future.

\subsection{Considerations about the method (simulations)}

One of the particularities of this study was its use of PBRs as shading elements for an outdoor work environment as an extension of a public library. In order to accomplish this, the daylighting performance of the PBRs had to be analyzed for a context that has a lack of reference parameters, specifically those for thoroughly assessing visual comfort conditions in outdoor workspaces. For instance, to the authors' knowledge, there is no formal specification for the maximum illuminance on a covered terrace to guarantee a perceived level of visual comfort for the occupants, as the requirements are typically set for an indoor space of a library (target workplane illuminance $=300 \mathrm{~lx}$ [49]). For this reason, the simulations were run using two upper thresholds:

- Maximum illuminance $=3000 \mathrm{~lx}$, taken from the studies carried out by Mardaljevic et al. [54] and used to set the upper limit of the UDI metric for indoor spaces. Illuminance values of over $3000 \mathrm{~lx}$ are likely to cause glare, and the value was proven to correlate reasonably well with more specific metrics for glare, such as the Daylight Glare Probability (DGP) [55].

- Maximum illuminance $=6000 \mathrm{~lx}$, to supplement the previous criterion, intended for indoor spaces, with one that accounted for the fact that higher illuminance levels are present in outdoor spaces, and the human visual system tends to adapt to 
such conditions. The value of $6000 \mathrm{~lx}$ was chosen based on a series of experimental campaigns carried out by one of the authors, which investigated how the livingscape (consisting of lightscape and soundscape) was subjectively perceived and assessed by users of outdoor spaces in two districts in Turin [56-58]. Furthermore, this upper threshold was found to be in good agreement with the findings from a study from Compagnon and Goyette-Pernot [59,60]: in the frame of the RUROS project, they investigated the visual comfort in two public spaces in Fribourg (Suisse), through both measurements and subjective surveys, and derived a correlation between the percent of people who reported a glare sensation with the cylindrical illuminance measured in-situ (this metric being preferable with respect to the horizontal illuminance). The finding show that less than $10 \%$ of respondents reported glare when the cylindrical illuminance was around $5000 \mathrm{~lx}$, this percent raising up to $40 \%$ for a cylindrical illuminance of 250 $\mathrm{klx}$ (direct sunlight). The above mentioned study from Torres and Lo Verso [55] show that, compared to the DGP, the thresholds for the horizontal illuminance and the cylindrical illuminance were comparable. It seems therefore meaningful that the threshold of $6000 \mathrm{~lx}$ assumed for the horizontal illuminance is somewhat comparable to the threshold of 5000 lx reported for the cylindrical illuminance in the study from Compagnon and Goynette-Pernot.

According to the authors' intent, both thresholds could be used as reference. When in the presence of an overcast sky condition, the range 300-3000 lx seems to be the most appropriate target, as the external unobstructed illuminance is typically lower than $15000 \mathrm{~lx}$ (for the time-steps considered in the study). On the other hand, in the presence of a clear sky condition, the range 300-6000 lx seems more appropriate, as the external illuminance is of course much higher (up to $65000 \mathrm{~lx}$ ). This means that allowing for a higher illuminance level under the PBR roof, in such a case would yield a lower illuminance contrast with the surrounding areas. Moreover, it should be noted that the PBRs installed to shade the outdoor workspace are translucent materials, with the consequence that the luminous environment generated inside the space is mainly diffused, also under a clear sky. Consequently, the luminance contrasts are smoothened and therefore discomfort is prevented for the occupants of the space. It also should be acknowledged that people tend to behave according to an adaptive comfort, thus accepting higher illuminance values in an outdoor space (compared to an indoor space), which is immersed in direct sunlight and skylight. Finally, it is worth stressing the positive impact expected of the presence of large PBRs with microalgae on people acceptance, in line with studies that investigate and demonstrate how façade elements and their interaction with light can influence occupant subjective and physiological responses [61].

Consistently, the simulations were run to obtain two different types of output.

- Point-in-time illuminances, for significant days of the year (the fall equinox and the two solstices) at two different times (9 in the early morning and 15 in the early afternoon), under opposite sky conditions (clear and overcast). During this phase of the analysis, among the three time-steps, particular attention was paid by the design team to September 21 at 9 and at 15 , in accordance with the prescriptions of the LEED protocol [51], which were used as initial guidelines for this phase.
Daylight Autonomy and Useful Daylight Illuminance (UDI ${ }_{300-}$ 3000 and $\left.\mathrm{UDI}_{300-6000}\right)$ to assess the daylighting performance of the PBRs on an annual basis. For this phase, the metrics prescribed in the latest LEED protocol [50,51], that are the spatial Daylight Autonomy $\mathrm{sDA}_{300,50 \%}$ and the Annual Sunlight Exposure $\mathrm{ASE}_{1000,250 \mathrm{~h}}$ were not used, as they were not entirely suitable for the analysis of an outdoor environment. The $\mathrm{sDA}_{300,50 \%}$ is defined as "the percent of an analyzed area that meets a minimum daylight illuminance level of $300 \mathrm{~lx}$ for $50 \%$ of the operating hours per year". It was not used in the study as it specifically refers to indoor spaces: moreover, due to the speculative nature of the illuminance threshold (3000 lx or $6000 \mathrm{~lx}$ ) the information would not be useful in assessing the daylighting performance of the PBRs. The DA was considered as more useful for the characterization of the external space. Differently, the $\mathrm{ASE}_{1000,250 \mathrm{~h}}$ is defined as the "percent of an analyzed area that exceeds a specified direct beam sunlight illuminance level of 1000 lx for more than 250 hours per year". This metric was not considered as it is intended for use in the absence of a shading system, to inform designers on how much direct sunlight reaches an opening and help them understand if a shading element is needed. In the case of the terrace, it is clear that the space is overexposed to direct sunlight and shading is needed.

\subsection{Innovations and limits of the study}

The key innovation in this study was the development of a new type of static PBR panel, capable of combining the advantages of two distinct types of PBR systems; the flat-panel type and the tubular-raceway type systems. The flat-panel type is typically comprised of a static vertical element, which employs an airlift system to achieve culture mixing. The latter tubular raceway type system, generally consists of a continuous tubular element distributed across a horizontal or inclined plane, and achieves mixing by circulating the culture medium. Tubular systems have been implemented successfully in horizontal and inclined configurations [11]; however they have disadvantages in their application as screening elements due to their refractory properties and the spacing of the tubular elements, which results in undesirable environmental lighting conditions. The solution proposed in this study aims to combine the luminous transmission properties of flat panel systems, with the mass transfer efficiency of tubular systems [11]. This was conceived in the form of corrugated flat panel elements, which cause the microalgal solution to flow in a similar way as in the tubular systems, while eliminating the disadvantages posed by gaps and light refraction. By setting the focus primarily on daylighting issues, the study is limited in that it does not account for thermal and ventilation conditions. On the other hand, it is worth stressing that the PBRbased shading system that was developed has the capacity to screen direct sunlight, admitting only diffuse daylight into the workspace below. Furthermore, the space is open along the sides, which allows natural ventilation to occur. Additionally, the illuminance values assumed as upper threshold for the operational conditions (3000 lx and $6000 \mathrm{~lx}$ ) assure that an excessive amount of sunlight is not entering the space. In light of these considerations however, a detailed thermal analysis will be the object of a future, dedicated study.

The design of the flat panel system and the circulation of the microalgae culture inside PBR was not developed beyond the concept stage in this study, and a homogeneous distribution of 
microalgae was assumed. This could be a further limitation due to the potential for biomass adhesion and sedimentation, which could play a role despite all efforts in culture mixing strategies. The gasliquid separation could be another issue that may be solvable through a dedicated sizing strategy. Studies on laboratory scale panels and CFD simulations will be the object of yet another future study.

\section{Conclusions}

A case study on the application of a shading system consisting of PBR with micro-algae is presented in the paper. It was developed as a preliminary design intended to take advantage of the opportunities presented by an existing public spaces, namely the building called 'the Edge', which belongs to the State Library of Queensland (SLQ) in Brisbane. Particularly, the shading system was defined for an outdoor multi-purpose workspace, located on the landscape roof terrace of the building. The proposed shading system was conceived to be responsive, giving adequate visual comfort levels for the occupants, despite the exposure to direct sunlight and fluctuating weather conditions. The paper has two main goals: $(i)$ to describe the application of the specifically designed PBR system; (ii) to assess the daylight performance of the outdoor space where the PBR system is installed.

As far as the first goal is concerned, a PBR with micro-algae was selected as responsive shading system to shade the landscape terrace of the Library, due to its sustainability in terms of trade-off between aesthetical issues, environmental comfort assured in the space and final acceptance for the occupants, in the line of 'biomimetic architecture'.

The resulting shading system consist of an array of photobioreactors (PBRs) containing micro-algae, positioned both on the roof and on the walls of the outside multipurpose workspace. The micro-algae cultivated within the shading elements belong to the Scenedesmus species: this species was chosen following earlier experimental studies that were carried out by the authors [4,5]. The microalgae contribute to improving the environment by biosequestrating $\mathrm{CO}_{2}$ and producing oxygen. They are also a valuable raw material containing bioactive compounds with various uses including nutraceutical products, livestock feed, and biofuel synthesis. In addition, their green pigmentation is visually engaging and allows for the filtration of solar radiation, with an expected positive acceptance from the occupants. The biomass density, and hence light transmittance, of the PBRs can be adjusted though a system control and an automated system in response to external daylighting, in such a way to guarantee an adequate level of visual comfort for the work environment that is exposed to direct sunlight and fluctuating weather conditions. In more detail, the biomass density within the PBRs is adjusted to provide a wide range of light transmittances (in the $\mathrm{T}_{\mathrm{V}}=10 \%-80 \%$ range) to counter the highly variable outdoor conditions (in the range 0 $15000 \mathrm{~lx}$ in overcast sky conditions, 15000-68000 lx in clear sky conditions).

As far as the second goal is concerned, the daylighting performances in the workspace was analysed by calculating the distribution of illuminance values inside the workspace using the simulation tool DIVA-for-Rhino, for different values of $\mathrm{T}_{\mathrm{v}}$. The illuminance values were used for two types of analyses: point-intime illuminances for specific time-steps during the course of a year (the two solstices and the Fall equinox); and annual determination of specific climate-based daylighting metrics such as the Daylight Autonomy $\left(\mathrm{DA}_{>300}, \mathrm{DA}_{>3000}\right.$, and $\left.\mathrm{DA}_{>6000}\right)$ from which the Useful Daylight Illuminance (UDI $\mathrm{UD0}_{30000}$ and $\left.\mathrm{UDI}_{300-6000}\right)$ was derived, according to two comfort ranges: 300-3000 $1 \mathrm{x}$ ('optimal' range) and 300-6000 lx ('acceptable' range). As a further step, the daylighting performances were compared using two other climates (Turin, Italy, and Dubai, United Arab Emirates), in order to verify the system's performance under various environmental conditions.

In short, the following conclusions can be drawn from the findings of the study:

- The choice of using a PBR with micro-algae as shading system was defined to be a suitable strategy to shade the outdoor multipurpose workplane of the 'Edge' building. The most suitable technology was chosen, which led to define a solution that used a hybrid configuration of tubular and flat-panel types in the form of a corrugated flat-panel. The final configuration of the PBR shading system was made up of horizontal and vertical panels, whose main components are transparent panes that serve to expose the culture medium to sunlight;

- A sustainable design strategy was developed, based on both a water strategy, aimed at exploiting the rainwater for both the library facilities and the PBR system, and an electricity strategy, based on photovoltaic cells. The surplus biomass produced by the PBR system is removed for processing offsite while the resulting biofuel is then returned to the system to produce electricity and heat;

- A supporting structure was specifically devised, consisting of columns, to be cast individually using a system of molds made from $\mathrm{CNC}$ machined foam blocks, and screening element, to be cast as a perforated shell encasing the column tops and consolidating the support structure;

- The amount of daylight transmitted to the workspace can be modified by adjusted the biomass density of the culture medium circulating within the panels. By increasing the biomass density, more daylight is absorbed along the light path resulting in less light exiting the panel, and vice versa. To do so, the circulation of biomass is autonomously controlled by monitoring software, which combines input from an array of photosensors with weather forecast data;

- The overall system is intended to be replicated and applied to other real buildings in other climates (the study also analysed the daylighting performances that could be obtained in Turin, Italy, and Dubai, United Arab Emirates);

- The daylighting results from both the PIT illuminance and UDI simulations showed that for clear sky conditions, the maximum $\mathrm{T}_{\mathrm{v}}$ of PBRs results is in the range 5\%-20\% to remain in the 'optimal' comfort range, and in the range $20 \%-30 \%$ to remain in the 'acceptable' comfort range. For overcast sky conditions, the maximum $\mathrm{T}_{\mathrm{v}}$ of PBRs results in the range $40 \%-80 \%$ to remain in the 'optimal' comfort range, and can be set to the maximum value of $80 \%$ to remain in the 'acceptable' comfort range;

- Taking all the climates into account (Brisbane, Turin, Dubai), moderate levels of biomass saturation are needed $\left(\mathrm{T}_{\mathrm{v}}<20 \%\right.$ for all the sites considered to remain in the 300-6000 $\mathrm{lx}$ illuminance comfort range), with higher concentrations $\left(\mathrm{T}_{\mathrm{v}}<\right.$ $10 \%$ ) to remain within the 300-3000 lx illuminance comfort range. Differently, in overcast sky conditions, the optimal 
range of illuminance is easily obtained with reduced levels of biomass saturation $\left(\mathrm{T}_{\mathrm{v}}<80 \%\right)$.

\section{Contributions}

Valerio R.M. Lo Verso played the major role in the development of the methodology for the daylighting simulations. He also carried out the daylighting analyses from the simulation results. Finally, he wrote the text of the paper, in cooperation with Matin H.S. Javadi.

Matin H. S. Javadi conceived and developed the idea and carried out most of studies on the state of the art and the literature review. He played the major role in the definition of the PBR system and its elements. He also created the 3D models and performed the daylighting simulations using DIVA-for-Rhino. Finally, he contributed to the writing of the text the paper, in cooperation with Valerio R.M. Lo Verso.

Simonetta Pagliolico played the major role in the analyses and characterization of the life cycle and biological properties of the algae in the BPR system. Furthermore, she cooperated in the synergic phase of the interpretation of the results.

Corrado Carbonaro played a crucial role in defining the feasibility and technological characterization of the PBR systems.

Guido Sassi actively cooperated in the definition of the project and in the interpretation of the results, especially by carrying out a sensitive analysis of the outcome.

\section{References}

[1] Carlorattiassociati, DIGITAL (SUB) TROPICAL Algaetecture - project: ecoLogicStudio in collaboration with MIT Senseable City Lab, Carlo Ratti, Giovanni de Niederhausern (project leader), Available at: https://carloratti.com/project/digital-sub-tropical/.

[2] K.-H. Kim, A Feasibility Study of an Algae Façade System. In: Conference 'SB13 Seoul - Sustainable Building Telegram toward Global Society', 2013, July 7-10, Seoul, Korea, Rotterdam (Netherlands) In-house publishing 2013:323-331.

[3] J. Wurm, The SolarLeaf bio-responsive façade: The first pilot project "BIQ" in Hamburg, Germany, In: Proceedings of "GlassCon Global - Innovation in Glass Technology', Philadelphia, PA, 2014, USA.

[4] S.L. Pagliolico, V.R.M. Lo Verso, F. Bosco, C. Mollea, C. La Forgia, A Novel Photo-bioreactor Application for Microalgae Production as a Shading System in Buildings, Energy Procedia 111 (2017) 151-160.

[5] S.L. Pagliolico, V.R.M. Lo Verso, M. Zublena, L. Giovannini, Preliminary results on a novel photo-bio-screen as a shading system in a kindergarten: Visible transmittance, visual comfort and energy demand for lighting, Solar Energy 185 (2019) 41-58.

[6] P. Chelf, L.M. Brown, C.E. Wyman, Aquatic biomass resources and carbon dioxide trapping, Biomass and Bioenergy 4 (1993) 175-183.

[7] C.H. Tan, P.L. Show, J.S. Chang, T.C. Ling, J.W.C. Lan, Novel approaches of producing bioenergies from microalgae: A recent review, Biotechnology Advances 33 (2015) 1219-1227.

[8] V. Mimouni, L. Ulmann, V. Pasquet, M. Mathieu, L. Picot, G. Bougaran, J.-P. Cadoret, A. Morant-Manceau, B. Schoefs, The Potential of Microalgae for the Production of Bioactive Molecules of Pharmaceutical Interest, Current Pharmaceutical Biotechnology 13(15) (2012) 2733-2750.

[9] F.J. Barba, N. Grimi, E. Vorobiev, New Approaches for the Use of Nonconventional Cell Disruption Technologies to Extract Potential Food Additives and Nutraceuticals from Microalgae, Food Engineering Reviews 7 (2015) 45-62.

[10] J.B.K. Park, R.J. Craggs, A.N. Shilton, Wastewater treatment high rate algal ponds for biofuel production, Bioresource Technology 102 (2011) 35-42.

[11] G. Olivieri, P. Salatino, A. Marzocchella, Advances in photobioreactors for intensive microalgal production: configurations, operating strategies and applications, Journal of Chemical Technology \& Biotechnology 89 (2014) 178-195.

[12] R.L. Chapman, Algae: the world's most important "plants"-an introduction, Mitigation and Adaptation Strategies for Global Change 18 (2013) 5-12.
[13] D. Hanelt, K. Bischof, K. Dunton, Life Strategy, Ecophysiology and Ecology of Seaweeds in Polar Waters, Reviews in Environmental Science and Bio/Technology 6 (2007) 95-126.

[14] A. Bahadar, M.B. Khan, Progress in Energy from Microalgae: A Review, Renewable \& Sustainable Energy Reviews 27 (2013) 128-148.

[15] P. Spolaore, C. Joannis-Cassan. E. Duran, A. Isambert, Commercial applications of microalgae, Journal of Bioscience and Bioengineering 101 (2006) 87-96.

[16] J.J. Milledge, Commercial application of microalgae other than as biofuels: a brief review, Reviews in Environmental Science and Bio/Technology 10 (2011) 31-41.

[17] S.A. Razzak, M.M. Hossain, R.A. Lucky, A.S. Bassi, Integrated CO2 capture, wastewater treatment and biofuel production by microalgae culturing. A Review, Renewable \& Sustainable Energy Reviews 27 (2013) 622-653.

[18] S. Wilkinson, P. Stoller, P. Ralph, B. Hamdorf, L.N. Catana, G.S. Kuzava, Exploring the feasibility of algae building technology in NSW, Procedia engineering 180 (2017) 1121-1130.

[19] V. Bhola, F. Swalaha, R. Ranjith Kumar, M. Singh, F. Bux, Overview of the potential of microalgae for $\mathrm{CO} 2$ sequestration, International Journal of Environmental Science and Technology 11 (2014) 2103-2118.

[20] E. Negev, A. Yezioro, M. Polikovsky, A. Kribus, J. Cory, L. Shashua-Bar, A. Golberg, Algae Window for reducing energy consumption of building structures in the Mediterranean city of Tel-Aviv, Israel, Energy and Buildings 204 (2019) 109460.

[21] B. Wang, C.Q. Lan, M. Horsman, Closed photobioreactors for production of microalgal biomasses, Biotechnology Advances 30 (2012) 904-912.

[22] P. Lavens, P. Sorgeloos, FAO fisheries technical paper 361, 1996, Ghent: University of Ghent.

[23] M. Talaei, M. Mahdavinejad, R. Azari, Thermal and energy performance of algae bioreactive façades: A review, Journal of Building Engineering 28 (2019) 101011.

[24] M.A. Ismail, K.M. Al-Obaidi, Exploration in Using Algae to Enhance Indoor Environment in the Tropical Climate, in: 2019 2nd Asia Conference on Energy and Environment Engineering (ACEEE), 2019, pp. 25-29, Japan.

[25] S. Hemming, A. Sapounas, W. Voogt, Algenteeltsystemen voor de tuinbouw - integratie, Wageningen: Wageningen UR (in Dutch), Wageningen University \& Research, Report GTB-1221, 2012.

[26] L. Travieso, D.O. Hall, K.K. Rao, F. Benitez, E. Sanchez, R. Borja, A helical tubular photobioreactor producing Spirulina in a semicontinuous mode, International Biodeterioration \& Biodegradation 47 (2001) 151-155.

[27] A. Richmond, The challenge confronting industrical microagriculture: high photosynthetic efficiency in large-scale reactors, Hydrobiologia 151 (1987) $117-121$.

[28] Y. Watanabe, D.O. Hall, Photosynthetic CO2 fixation technologies using a helical tubular bioreactor incorporating the filamentous cyanobacterium Spirulina platensis, Energy Conversion and Management 36 (1995) 721724.

[29] A. Contreras, F. Garcia, E. Molina, J. Merchuk, Interaction between CO2mass transfer, light availability, and hydrodynamic stress in the growth of Phaeodactylum tricornutum in a concentric tube, Biotechnology and Bioengineering 60 (1998) 317-325.

[30] Q.H. Hu, H. Guterman, A. Richmond, A flat inclined modular photobioreactor for outdoor mass cultivation of photoautotrophs, Biotechnology and Bioengineering 51 (1996) 51-60.

[31] N. Zou, A. Richmond, Effect of light-path length in outdoor flat plate reactors on output rate of cell mass of EPA in Nannochloropsis sp, Progress in Industrial Microbiology 35 (1999) 351-356.

[32] M.A. Borowitzka, Commercial production of microalgae: ponds, tanks, tubes and fermenters, Journal of Biotechnology 70 (1999) 313-21.

[33] O. Zmora, A. Richmond, Microalgae for aquaculture: microalgae production for aquaculture, In: Handbook of microalgal culture: Biotechnology and Applied Phycology, Amos R. editor, 2007, pp. 365-379.

[34] E. Sierra, F.G. Acién, J.M. Fernández, J.L. García, C. González, E. Molina, Characterization of a flat plate photobioreactor for the production of microalgae, Chemical Engineering Journal 138 (2008) 136-147.

[35] F. Martínez-Jerónimo, F. Espinosa-Chávez, A laboratory-scale system for mass culture of freshwater microalgae in polyethylene bags, Journal of Applied Phycololgy 6 (1994) 423-425.

[36] D. Hill. Post-occupancy evaluation of wi-fi, State Library of Queensland, 2009, 205624-00. Available at: https://www.cityofsound.com/blog/2008/08/post-occupancy.html, Retrieved on: October 2019.

[37] J. Baehr-Bruyère, K. Chamilothori, A.P. Vassilopoulos, J. Wienold, M. Andersen, Shaping light to influence occupants' experience of space: a 
kinetic shading system with composite materials, Journal of Physics: Conference Series 1343 (2019) 012162.

[38] L. Giovannini, V.R.M. Lo Verso, B. Karamata, M. Andersen, Lighting and Energy Performance of an Adaptive Shading and Daylighting System for Arid Climates, Energy Procedia 78 (2015) 370-375.

[39] S. Iennarella, V. Serra, V.R.M. Lo Verso, A novel concept of a responsive transparent façade module: optimization of energy performance through parametric design, Energy Procedia 78 (2015) 358-363.

[40] A.M. Al-Masrani, K.M. Al-Obaidi,Dynamic shading systems: A review of design parameters, platforms and evaluation strategies, Automation in construction 102 (2019) 195-216.

[41] K.M. Al-Obaidi, M.A. Ismail, H. Hussein, A.M.A. Rahman, Biomimetic building skins: An adaptive approach, Renewable and Sustainable Energy Reviews 79 (2017) 1472-1491.

[42] K.M Dewidar, N.M. Mohamed, Y.S. Ashour, Living Skins: A New Concept of Self Active Building Envelope Regulating Systems, in: International Conference Sustainable Building SB13, paper 28, 2013, Dubai.

[43] C.M. Palmer, A composite rating of algae tolerating organic pollution, Journal of Psychology 5 (1969) 78-82.

[44] S.L. Pagliolico, V.R.M. Lo Verso, A. Torta, M. Giraud, F. Canonico, L. Ligi, A preliminary study on light transmittance properties of translucent concrete panels with coarse waste glass inclusion, Energy Procedia 78 (2015) 1811-1816.

[45] M. El-Sheekh, A. El-Fatah Abomohra, H. Dieter, Optimization of Biomass and Fatty Acid Productivity of Scenedesmus Obliquus as a Promising Microalga for Biodiesel Production, World Journal of Microbiology and Biotechnology 29 (2013) 915-922.

[46] C. F. Reinhart, J. Mardaljevic, Z. Rogers, Dynamic daylight performance metrics for sustainable building design, LEUKOS 3 (2006) 7-31.

[47] A. Nabil, J. Mardaljevic, Useful Daylight Illuminance: a new paradigm to access daylight in buildings, Lighting Research and Technology 37 (2005) $41-59$.

[48] A. Nabil and J. Mardaljevic, Useful daylight illuminances: A replacement for daylight factors, Energy and Buildings 38 (2006) 905-913.

[49] CEN (Comité Européen de Normalisation), European Standard EN 124641:2011, Light and lighting - Lighting of work places - Part 1: Indoor work places, 2011, Brussels.

[50] IES Daylight Metrics Committee, IES Spatial Daylight Autonomy (sDA) and Annual Sunlight Exposure (ASE), Daylight Metrics Committee. Approved Method IES LM-83-12, Illuminating Engineering Society of North America, 2012.
[51] USGBC (United States Green Building Council). LEED Reference Guide for Building Design and Construction (v4), US Green Building Council, 2019.

[52] CEN (Comité Européen de Normalisation), European Standard EN 15193 1:2017, Energy performance of buildings - Energy requirements for lighting - Part 1: Specifications, Module M9, 2017, Brussels.

[53] R. Pagani, C. Carbonaro, L. Savio, C. Boonstra, E. De Oliveira Fernandes. Concerto Al Piano. Sustainable Urban Transformations. Franco Angeli editor (2016) 1-171.

[54] J. Mardaljevic, M. Andersen, N. Roy, J. Christoffersen, Daylighting metrics for residential buildings, In: 27th Session of CIE International Conference, Sun City, 2011, South Africa.

[55] S. Torres, V.R.M. Lo Verso, Comparative analysis of simplified daylight glare methods and proposal of a new method based on the cylindrical illuminance, Energy Procedia 78 (2015) 699-704

[56] F. La Malva, A. Astolfi, P. Bottalico, V.R.M. Lo Verso, F. Bronuzzi, Livingscape approach to improve urban historical places, Journal of Temporal Design (2011), 20-24.

[57] F. La Malva, A. Astolfi, P. Bottalico, V.R.M. Lo Verso, F. Bronuzzi, Livingscape multi-sensory experience in urban historical places: subjective assessment from the local people and quality of the urban environment, Journal of Temporal Design in Architecture and the Environment 11 (2011) 25-29.

[58] F. La Malva, A. Astolfi, V.R.M. Lo Verso, Livingscape: a multi-sensory approach to improve the quality of urban spaces, Energy Procedia 78 (2015) 37-42.

[59] J. Goyette, R. Compagnon, Rediscovering the Urban Realm and Open Space Project: two case studies in Fribourg, Switzerland, In: ICUC-5 Fifth International Conference on Urban Climate, 2003, Lodz, Poland.

[60] R. Compagnon, J. Goyette-Pernot, Visual Comfort in urban spaces (in Italian): Chapter in: (cured by) A. Rogora, V. Dessì. Environmental comfort in open outdoor spaces (in Italian), Edicom Edizioni, Montfalcone (Gorizia), 2005.

[61] K. Chamilothoria, G. Chinazzo, J. Rodrigues, E.S. Dan-Glauserc, J. Wienold, M. Andersen, Subjective and physiological responses to façade and sunlight pattern geometry in virtual reality, Building and Environment 50 (2019) 144-155. 